\title{
Regex Matching with Counting-Set Automata
}

\author{
LENKA TUROŇOVÁ* ${ }^{*}$ Brno University of Technology, Czech Republic \\ LUKÁŠ HOLÍK, Brno University of Technology, Czech Republic \\ ONDŘEJ LENGÁL, Brno University of Technology, Czech Republic \\ OLLI SAARIKIVI, Microsoft, USA \\ MARGUS VEANES, Microsoft, USA \\ TOMÁŠ VOJNAR, Brno University of Technology, Czech Republic
}

We propose a solution to the problem of efficient matching regular expressions (regexes) with bounded repetition, such as (ab) $\{1,100\}$, using deterministic automata. For this, we introduce novel counting-set automata (CsAs), automata with registers that can hold sets of bounded integers and can be manipulated by a limited portfolio of constant-time operations. We present an algorithm that compiles a large sub-class of regexes to deterministic CsAs. This includes (1) a novel Antimirov-style translation of regexes with counting to counting automata (CAs), nondeterministic automata with bounded counters, and (2) our main technical contribution, a determinization of CAs that outputs CsAs. The main advantage of this workflow is that the size of the produced CsAs does not depend on the repetition bounds used in the regex (while the size of the DFA is exponential to them). Our experimental results confirm that deterministic CsAs produced from practical regexes with repetition are indeed vastly smaller than the corresponding DFAs. More importantly, our prototype matcher based on CsA simulation handles practical regexes with repetition regardless of sizes of counter bounds. It easily copes with regexes with repetition where state-of-the-art matchers struggle.

CCS Concepts: • Theory of computation $\rightarrow$ Regular languages; Quantitative automata; $\bullet$ Security and privacy $\rightarrow$ Denial-of-service attacks; • Applied computing $\rightarrow$ Document searching.

Additional Key Words and Phrases: regular expression matching, bounded repetition, ReDos, determinization, Antimirov's derivatives, counting automata, counting-set automata

ACM Reference Format:

Lenka Turoňová, Lukáš Holík, Ondřej Lengál, Olli Saarikivi, Margus Veanes, and Tomáš Vojnar. 2020. Regex Matching with Counting-Set Automata. Proc. ACM Program. Lang. 4, OOPSLA, Article 218 (November 2020), 30 pages. https://doi.org/10.1145/3428286

\section{INTRODUCTION}

Matching regexes (regular expressions) is a ubiquitous component of software, used, e.g., for searching, data validation, parsing, finding and replacing, data scraping, or syntax highlighting. It

\footnotetext{
*The main part of the work was done when the first author was a summer intern at Microsoft Research in Redmond in 2019.

Authors' addresses: Lenka Turoňová, Faculty of Information Technology, Brno University of Technology, Božetěchova 2 , Brno, 612 00, Czech Republic, ituronova@fit.vutbr.cz; Lukáš Holík, Faculty of Information Technology, Brno University of Technology, Božetěchova 2, Brno, 612 00, Czech Republic, holik@fit.vutbr.cz; Ondřej Lengál, Faculty of Information Technology, Brno University of Technology, Božetěchova 2, Brno, 612 00, Czech Republic, lengal@fit.vutbr.cz; Olli Saarikivi, MSR, Microsoft, One Microsoft Way, Redmond, 98052, USA, Olli.Saarikivi@microsoft.com; Margus Veanes, MSR, Microsoft, One Microsoft Way, Redmond, 98052, USA, margus@microsoft.com; Tomáš Vojnar, Faculty of Information Technology, Brno University of Technology, Božetěchova 2, Brno, 612 00, Czech Republic, vojnar@fit.vutbr.cz.
}

This work is licensed under a Creative Commons Attribution 4.0 International License.

(c) 2020 Copyright held by the owner/author(s).

2475-1421/2020/11-ART218

https://doi.org/10.1145/3428286

Proc. ACM Program. Lang., Vol. 4, No. OOPSLA, Article 218. Publication date: November 2020. 
is commonly used and natively supported in most programming languages [contributors 2019]. For instance, about 30-40\% of Java, JavaScript, and Python software use regex matching (as reported in multiple studies, see, e.g., [Davis 2019]).

The efficiency of regex matching engines has a significant impact on the overall usability of software applications. Unpredictability of a matcher's performance may lead to catastrophic consequences, witnessed by events such as the recent catastrophic outage of Cloudflare services [GrahamCumming 2019], caused by a single poorly written regex, and it is a doorway for the so-called ReDoS attack, a denial of service attack based on overwhelming a regex matching engine by providing a specially crafted regex or text. For instance, in 2016, ReDoS caused an outage of StackOverflow [Exchange 2016] or rendered vulnerable websites that used the popular Express.js framework [Baldwin 2016]. Works such as [Davis 2019; Davis et al. 2018] give arguments that ReDoS is not just a niche problem but rather a common and serious threat.

Failures of matching are mostly caused by the so-called "catastrophic backtracking", a situation when variants of Spencer's simulation of a nondeterministic finite automaton (NFA) by backtracking [Spencer 1994] exhibit a behaviour super-linear to the length of the text. Matching algorithms based on backtracking are probably the most often implemented ones, their performance is, however, at worst exponential to the text length. An alternative with a much lower worst-case complexity (wrt the length of the text) is to use deterministic finite automata (DFAs). In the ideal case, the DFA is precomputed; matching can then be linear to the text length, with each input symbol processed in constant time. This is the so-called static DFA simulation [Sipser 2006]. The major drawback of static DFA simulation is that the DFA construction may explode, rendering the method unusable in practice.

Variants of Thompson's algorithm [Thompson 1968] (sometimes called NFA simulation or NFA-toDFA simulation) avoid the explosion by working directly with the NFA. They essentially run the determinization by subset construction on the fly, always remembering only the current DFA state. On reading a character, a successor DFA state is computed and used to replace the current state. The disadvantage of Thompson's algorithm is that, for a highly nondeterministic NFA, the DFA states-sets of the states of the NFA-may get large and computing a DFA-state successor over a symbol becomes expensive, linear to the size of the NFA (compared to the constant time of static DFA simulation).

Modern matchers therefore use caching of already visited parts of the DFA. Making a step within the cached part is then as fast as with the explicitly determinized automaton. Extremely efficient implementations of Thompson's algorithm with caching are used in RE2 [Google [n.d.]] and GNU grep [Haertel et al. [n.d.]]. Their close cousin, an on-the-fly Brzozowski's derivative construction, is implemented in the tool SRM [Saarikivi et al. 2019]. Highly nondeterministic regexes ${ }^{1}$ that lead to exploding determinization are, however, problematic for all variants, explicit determinization as well as NFA simulation, with or without caching.

In this paper, we focus on eliminating a frequent cause of a DFA explosion-a use of the counting operator, also known as the operator of bounded repetition. It succinctly expresses repeated patterns such as $(a b)\{1,100\}$, representing 1 to 100 consecutive repetitions of ab. Such expressions are very common (cf. [Björklund et al. 2015]), e.g., in the RegExLib library [RegExLib.com [n.d.]], which collects expressions for recognizing URIs, markup code, pieces of Java code, or SQL queries; in the Snort rules [M. Roesch et al. [n.d.]] used for detecting attacks in network traffic; in real-life XML schemas, with the counter bounds being as large as 10 million [Björklund et al. 2015]; or in detecting information leakage from traffic logs [Holík et al. 2019].

\footnotetext{
${ }^{1}$ Loosely speaking, a "highly nondeterministic regex" is one for which the determinization of the NFA created by some of the usual algorithms explodes. Determinism of regexes closely corresponds to the notion of 1-unambiguity of the regex [Brüggemann-Klein and Wood 1998; Hovland 2009]: when matching a text from left to right against the regex, it is always clear which letter of the regex matches the text character.
} 
To illustrate the principal difficulty with matching bounded repetitions, especially when combined with a high degree of nondeterminism, consider the regex $\bullet \star a_{\bullet}\{k\}$ where $k \in \mathbb{N}$ (the regex denotes strings where the symbol a appears $k$ positions from the end of the word). Already the NFA will have at least $k$ states, which is exponential to the regex size because $k$ is written in decimal. Due to the inherent nondeterminism of this regex, determinization then adds a second level of the exponential explosion. Indeed, the minimal DFA accepting the language has $2^{k+1}$ states because it must remember all the positions where the symbol a was seen during the last $k+1$ steps. This requires a finite memory of $k+1$ bits and thus $2^{k+1}$ reachable DFA states. Determinizing the NFA explicitly is thus out of question for even moderate values of $k$. The pure Thompson's NFA simulation is feasible but very slow, as reading each character may in the worst case require processing the entire NFA. Moreover, caching of the DFA state space, used in industrial matchers like RE2 [Google [n.d.]] or GNU grep [Haertel et al. [n.d.]], may also be ineffective due to the size of the state space and low cache utilization. At the same time, combinations of nondeterminism and counting are fairly common. A high degree of nondeterminism is, for instance, usual when searching for a pattern "anywhere on the line" (corresponding to prefixing the pattern with.$*$ ), which is the standard behaviour for GNU grep and similar programs when start/end of line anchors are not used.

To facilitate efficient matching of such nondeterministic counting, we propose a translation from regexes with repetition to deterministic machines that are succinct and can perform matching with nearly constant character complexity. The novel succinct and fast deterministic machine, called the counting-set automaton (CsA), is the key to the result. It is a deterministic finite automaton with a special type of registers that can hold values called counting sets-a set of bounded integer values-and support a limited selection of simple set operations. Crucially for the efficiency of our approach, we show that, using a suitable data structure, all the set operations can be implemented to run in constant time regardless of the size of the set.

Our compilation from regexes to CsAs proceeds in two steps. First, we compile the regexes into nondeterministic counting automata $(C A s)$, automata with counters whose values are a priori bounded. Variants of CAs have been used in several other works under different names, e.g., [Björklund et al. 2015; Gelade et al. 2012; Holík et al. 2019; Hovland 2009; Kilpeläinen and Tuhkanen 2007; Smith et al. 2008b; Sperberg-McQueen [n.d.]]. The compilation from regexes is cheap and produces automata whose size is independent of the counter bounds and linear in the size of the regex. We present a novel translation of regexes to CAs that generalizes the Antimirov's derivative construction [Antimirov 1996]. Our translation has several advantages over the existing alternatives, such as absence of $\epsilon$-transitions in the output CA and succinctness. The result of translating the regex $* * a .\{k\}$ into a CA is illustrated in Fig. 1a.

The main step forward we make in this paper is a solution of efficient matching for a large class of highly nondeterministic regexes with counting that are quite common in practice. The main technical problem we have solved is a succinct transformation of a (nondeterministic) CA into a deterministic CsA. Our algorithm produces a CsA in time independent of the counter bounds. We note that this has been a known open problem (emphasized, e.g., in [Sperberg-McQueen [n.d.]]) Works on matching of bounded repetition such as [Björklund et al. 2015; Gelade et al. 2012; Holík et al. 2019; Hovland 2009; Kilpeläinen and Tuhkanen 2003; Kilpeläinen and Tuhkanen 2007; Smith et al. 2008a] mostly focus on deterministic regexes and do not propose practical solutions for the nondeterministic case. We have carried out an extensive experimental evaluation of our algorithm on a large sample of regexes used for pattern matching in various applications. The experiments show that our algorithm, although also limited to a sub-class of regexes, handles over $90 \%$ of regexes with counting we collected. The obtained data confirm that our CsAs are indeed far smaller and can be constructed faster than corresponding DFAs. Most importantly, we demonstrate the practical 


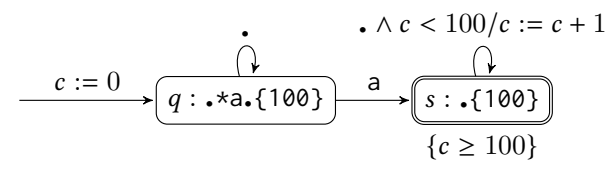

(a) The CA for the regex $\bullet \star a_{\bullet}\{k\}$

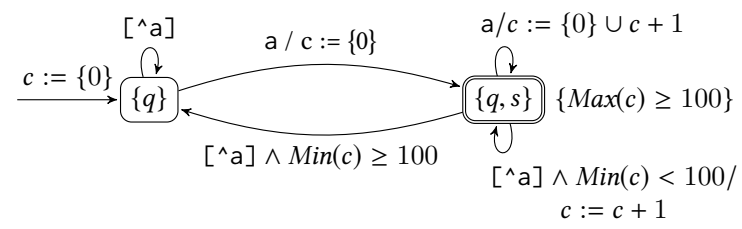

(b) The CsA from determinization of (a)

Fig. 1. The nondeterministic CA and the deterministic CsA for.$* a_{\bullet}\{100\}$. The transitions are labeled by their guard, which gives the character class (in the standard POSIX regex notation, where, e.g., • stands for "any character") and possibly restricts counter values, delimited by "/" from the counter update. If a counter does not have the update specified, then the transition does not change its value. In (b), the notation $c+1$ stands for the set of values obtained by incrementing each value in $c$ and then removing values larger than the upper bound 100 of the counter. The edges denoting initial states are labelled with initial values of the counters. Final states are labelled with an acceptance condition, e.g. $\{c \geq 100\}$ in (a).

The formal counter operations $\mathrm{OP}_{c}$ presented later in Section 4 are in (a) shown as follows: the guard of $\mathrm{OP}_{c}$ is shown in conjunction with the character guard $\alpha$ on the left of the "/", the update of $\mathrm{OP}_{c}$ is shown on the right of "/" in the form of an assignment to $c$, where $\mathrm{INCR}_{c}$ appears as the right value $c+1$, EXIT as 0 , EXIT1 as 1 , and NOOP is omitted.

relevance of our algorithm for pattern matching. We have implemented a matching algorithm based on CsA simulation ${ }^{2}$ and compared it with several state-of-the-art matchers, namely, grep [Haertel et al. [n.d.]], RE2 [Google [n.d.]], SRM [Saarikivi et al. 2019], and .NET [Microsoft 2020]. Our results show that problematic highly nondeterministic regexes with counting indeed appear in practice and can also be easily crafted as a ReDoS attack, and that CsAs can efficiently solve most of such problematic cases. For instance, the regex (_a) $\{64999\}$ _a from [Davis et al. 2019] can cause state-of-the-art matchers exceed any reasonable time limit (when searching for the pattern anywhere on the line, with the implicit $*$ in front). Already with the counter bounds lowered to 1,000 , the matchers take from 6 to 34 seconds on $500 \mathrm{KiB}$ of text, but our algorithm needs only 1 second even with the original bound 64,999.

We summarize the technical contributions of this work as follows:

(1) A novel Antimirov style regex-to-CA translation.

(2) A novel notion of the counting-set automaton, a deterministic machine that allows for succinct representation of counting constraints and fast matching.

(3) CA-to-CsA determinization that runs in time independent of counter bounds.

(4) A regex-matching algorithm interconnecting the above, efficient regardless of counter bounds especially on regexes that combine counting with nondeterminism.

(5) Implementation and extensive experimental evaluation of the above.

\section{OVERVIEW}

We give a brief overview of our conversion of a regex with counting into a deterministic CsA. We use the example regex $R=\bullet_{\star} \star a_{\bullet}\{100\}$, discussed already in the introduction and representing strings where the symbol a appears 100 positions from the end, with the corresponding minimum DFA having $2^{101}$ states. The conversion proceeds in two steps. First, $R$ is translated into a nondeterministic CA (Fig. 1a), denoted as CA $(R)$; second, $\mathrm{CA}(R)$ is converted into a deterministic CsA (Fig. 1b). The size of both is independent of the counter bounds (both of the automata will have 2 states only).

\footnotetext{
${ }^{2}$ We use a pre-computed deterministic CsA. While on-the-fly determinization is also possible, it was not needed in our experimentation since we have not witnessed problems with CsA state space explosion.
} 
Counting-Set Data Structure. Before looking into the conversion from regular expressions to CsAs it is useful to first understand why the resulting CsA can be used efficiently for matching in the first place. The main enabler behind this is the use of our counting-set data structure, say $c$, representing sets $S_{c} \subseteq\left\{0, \ldots, \boldsymbol{m a x}_{c}\right\}$ where the upper bound $\boldsymbol{m a x}_{\boldsymbol{c}}$ is a fixed positive integer. A runtime value of $c$ is a tuple $(o, \ell)$ where $o \in \mathbb{N}$ is called an offset and $\ell$ is a queue of strictly increasing natural numbers such that $S_{c}=\{o-n \mid n \in \ell\}$.

The data structure supports constant-time implementations of the following operations, assuming constant-time access to the first and the last element of the queue (the queue may be implemented as a doubly-linked list).

- The minimum and the maximum of $S_{c}$ are obtained as $o-\operatorname{last}(\ell)$ and $o-f i r s t(\ell)$, respectively.

- Insert 0: if $o-\operatorname{last}(\ell)>0$, then append $o$ at the end of $\ell$ (similarly for inserting 1).

- Increment all, up to $\max _{c}: o:=o+1$; if $o-f \operatorname{irst}(\ell)>\boldsymbol{m a x}_{c}$, then remove first $(\ell)$.

- Reset to $\{0\}: \ell:=0 ; o:=0$ (similarly for reset to $\{1\}$ ).

The independence of the running time of these operations of $\boldsymbol{m a x}_{c}$ enables our major achievement:

the independence of the running time of pattern matching of the counter bounds.

Let us now illustrate how this data structure works during matching. We run the CsA in Fig. 1b, assuming the meaning of the operations provided above, over the sample input word $a \theta^{(10)} \mathrm{aab}^{(87)} \mathrm{dfa}$. The configurations of the automaton after processing prefixes of the word are shown in the table: the control state, the

\begin{tabular}{llll} 
prefix & state & $(o, \ell)$ & $S_{c}$ \\
\hline$\epsilon$ & $\{q\}$ & $(0,[0])$ & $\{0\}$ \\
$\mathrm{a}$ & $\{q, s\}$ & $(0,[0])$ & $\{0\}$ \\
$\mathrm{aa}$ & $\{q, s\}$ & $(1,[0,1])$ & $\{1,0\}$ \\
$\mathrm{aa} \theta^{(10)}$ & $\{q, s\}$ & $(11,[0,1])$ & $\{11,10\}$ \\
$\mathrm{aa} \theta^{(10)} \mathrm{aa}$ & $\{q, s\}$ & $(13,[0,1,12,13])$ & $\{13,12,1,0\}$ \\
$\mathrm{aa} \theta^{(10)} \mathrm{aab}^{(87)}$ & $\{q, s\}$ & $(100,[0,1,12,13])$ & $\{100,99,88,87\}$ \\
$\mathrm{aa} \theta^{(10)} \mathrm{aab}^{(87)} \mathrm{d}$ & $\{q, s\}$ & $(101,[1,12,13])$ & $\{100,89,88\}$ \\
$\mathrm{aa} \theta^{(10)} \mathrm{aab}^{(87)} \mathrm{df}$ & $\{q, s\}$ & $(102,[12,13])$ & $\{90,89\}$ \\
$\mathrm{aa} \theta^{(10)} \mathrm{aab}^{(87)} \mathrm{dfa}$ & $\{q, s\}$ & $(103,[12,13,103])$ & $\{91,90,0\}$ \\
& & &
\end{tabular}
counting-set run-time configuration $(o, \ell)$, and the value $S_{c}$ it represents. The state $\{q, s\}$ fulfills the accepting condition after processing the 6 th and the 7 th prefix since the maximum of $S_{c}$ at these points is indeed at least 100 .

From a Nondeterministic CA to a Deterministic CsA. The idea of our CA-to-CsA determinization is best explained by comparison with the naive determinization of a CA, which would create a DFA by the explicit textbook-style subset construction. The states of the DFA would then be sets of runtime configurations of the $\mathrm{CA}$ where each CA-configuration would consist of a control state and a counter valuation. Counter valuations would hence be "unfolded"-they would become an explicit part of the DFA control states-and the succinctness provided by counters would be lost. For instance, the run of the CA in Fig. 1a on the word aa0 ... generates "powerstates"

$\{(q, c=0)\},\{(q, c=0),(s, c=0)\},\{(q, c=0),(s, c=0),(s, c=1)\},\{(q, c=0),(s, c=1),(s, c=2)\}, \ldots$ which are essentially subsets of $\{q, s\} \times\{0, \ldots, 100\}$. In the worst case, the size of the DFA would be exponential in counter bounds because $s$ can be paired with any subset of $\{0, \ldots, 100\}$ recording possible values of $c$. In contrast to this, as illustrated above, our CsA represents the counter valuations implicitly: it computes them dynamically on the fly and stores them as counting sets-i.e., the valuation of a counter changes from an integer to a set of integers. The counter valuations are hence not a part of control states, and their overall number influences neither the size of the CsA nor the time needed to build them.

Fig. $1 \mathrm{~b}$ shows the CsA obtained from determinization of the CA in Fig. 1a. The runtime configurations of the CsA reached for the word aa 0 are

$$
(\{q\}, c \in\{0\}), \quad(\{q, s\}, c \in\{0\}), \quad(\{q, s\}, c \in\{0,1\}), \quad(\{q, s\}, c \in\{1,2\}) .
$$


They encode the first three states reached by the sample DFA run above. Namely, the control states are kept in the first component and the counter values are in the second component, i.e., the set $S_{c}$ given by the run-time values of the counting-set $c$. In this encoding, the value of the counting-set is not relevant for the states where the counter is never active (state $q$ in our example). The counter's value in these states is always implicitly 0 . In the example, the value $S_{c}$ of the counting-set therefore only records the values of $c$ at state $s$ and is thus relevant only in the CsA state $\{q, s\}$. We note that for simplicity, we initialise all counting-sets uniformly with $\{0\}$, even if their value is initially irrelevant, as in the case of the CsA state $\{q\}$ in the example.

We note that some DFA powerstates cannot be encoded as CsA configurations due to the involved Cartesian abstraction: essentially, any state in the powerset is paired with any counter value from the counting set. Hence, our approach does not handle the full class of regexes. Fortunately, as our empirical evidence shows, regexes that fall out from the supported class are rare in practice.

From Regexes to Nondeterministic CAs. To translate a regex into a CA, we propose a generalization of the Antimirov's derivative construction [Antimirov 1996] to symbolic counting. In Antimirov's setting, a derivative of a regex $R$ wrt a character class $\alpha$ is a set of regexes that together capture all tails of words in $\mathcal{L}(R)$ whose head character is from $\alpha$. In particular, according to [Saarikivi et al. 2019], which generalizes [Antimirov 1996] to explicit counting, the derivatives of the regex $R=.^{*}$ a. $\{100\}$ wrt the classes a and [^a] are $\{R, .\{100\}\}$ and $\{R\}$, respectively. Further, for $1 \leq k \leq$ 100 , the derivative of $\bullet\{k\}$ wrt both $a$ and $[\wedge a]$ is $\{\bullet\{k-1\}\}$. The derivatives become the states of the resulting NFA, with $R$ being initial and.$\{0\}$ final, and with $\alpha$-transitions from each regex to all its $\alpha$-derivatives (for $\alpha$ being either a or [^a]). The obtained NFA is already large, it has 102 states.

In our new construction, the counting is kept implicit using symbolic counters. Instead of modifying the counter bound of the derivative (by, e.g., deriving $\bullet\{99\}$ from $\cdot\{100\}$ ), we keep the original bound unchanged and use a counter $c$ to keep track of the difference between the original value and the current value. Our conditional derivative operator $\partial_{\alpha}(\cdot)$ then equips the produced derivatives with conditional counter updates to keep the counters up-to-date. For instance, $\partial_{\mathrm{a}}(\cdot\{100\})$ returns the same regex $\cdot\{100\}$, but it is paired with conditional counter updates for $c$, namely, "if $c<100$, then increment $c$; and if $c \geq 100$, then exit the counting loop". The CA we obtain this way is shown in Fig. 1a, where the first conditional update translates to the self loop on the state.$\{100\}$ and the second to the acceptance condition. The size of the CA does not depend on the counter bounds.

\section{PRELIMINARIES}

We cast our definitions in the framework of symbolic automata [D'Antoni and Veanes 2020], a natural succinct representations of finite-state transition relations over large alphabets of labels. Symbolic automata work over alphabets equipped with a so-called effective Boolean algebra, which defines the needed interface for handling large sets of labels on automata transitions.

Before providing the formal definition of an effective Boolean algebra, we start with an intuitive example, which is also going to be the alphabet algebra used throughout the paper, including the experiments. Later on, we will further leverage the general definition to work with algebras over counter and counting-set predicates.

Example 3.1. Regular expressions in practice use character classes as basic building blocks. To simplify the discussion, let us restrict our attention to ASCII as the character universe $\mathfrak{D}$. In other words, $\mathcal{D}$ is the set $\left\{n \mid 0 \leq n<2^{7}\right\}$ of all 7-bit characters represented using their character codes. Then, for example, the character classes $[0-9]$ and $[A-Z]$ denote, respectively, the set $\llbracket[0-9] \rrbracket=\{n \mid 48 \leq n \leq 57\}$ of all digit codes, and the set $\llbracket[A-Z] \rrbracket=\{n \mid 65 \leq n \leq 90\}$ of all upper-case letter codes. Character classes made up of individual symbols such as a denote singleton sets, e.g., $\llbracket @ \rrbracket=\{64\}$. Character classes can also be used to form unions, they can be complemented, and even subtracted from each other, and are in general closed under Boolean 
operations. There are therefore many different ways how to represent the same character sets, e.g., $\llbracket[0-9] \rrbracket=\llbracket[0-45-9] \rrbracket=\llbracket[0-4] \rrbracket \cup \llbracket[5-9] \rrbracket$. To illustrate the complement, for example, $[\wedge \theta-9]$ denotes the set of all non-digits, as does $[\backslash \times 00-\backslash \times 2 F \backslash \times 3 A-\backslash \times 7 F]$. The set of all character classes is then an example of the set $\Psi$ of all predicates of a Boolean algebra, and checking satisfiability of a predicate $\varphi \in \Psi$ means to decide whether $\varphi$ denotes a nonempty set. For example, the predicate [] is unsatisfiable because $\mathbb{[}[] \rrbracket=\emptyset$ and . denotes the true predicate because $\mathbb{\|} \cdot \rrbracket=\mathfrak{D}$. Further, note that a character class can, without loss of generality, be represented as a Boolean combination of intervals or even as a union of intervals if normalized.

\subsection{Effective Boolean Algebras}

An effective Boolean algebra $\mathbb{A}$ has components $\left(\mathfrak{D}, \Psi, \mathbb{L} \_\mathbb{l}, \perp, \top, \vee, \wedge, \neg\right)$ where $\mathfrak{D}$ is a universe of underlying domain elements. $\Psi$ is a set of unary predicates closed under the Boolean connectives $\vee, \wedge: \Psi \times \Psi \rightarrow \Psi$ and $\neg: \Psi \rightarrow \Psi$; and $\perp, \top \in \Psi$ are the false and true predicates. Values of the algebra are sets of domain elements, and the denotation function $\mathbb{I} \_\mathbb{\|}: \Psi \rightarrow 2^{\mathfrak{D}}$ satisfies that $\llbracket \perp \rrbracket=\emptyset, \llbracket \top \rrbracket=\mathfrak{D}$, and for all $\varphi, \psi \in \Psi, \llbracket \varphi \vee \psi \rrbracket=\llbracket \varphi \rrbracket \cup \llbracket \psi \rrbracket, \llbracket \varphi \wedge \psi \rrbracket=\llbracket \varphi \rrbracket \cap \llbracket \psi \rrbracket$, and $\llbracket \neg \varphi \rrbracket=\mathfrak{D} \backslash \llbracket \varphi \rrbracket$. For $\varphi \in \Psi$, we write $\operatorname{Sat}(\varphi)$ when $\llbracket \varphi \rrbracket \neq \emptyset$, and we say that $\varphi$ is satisfiable. We require that Sat as well as $\vee, \wedge$, and $\neg$ are computable as a part of the definition of an effective Boolean algebra. We write $x=\varphi$ for $x \in \llbracket \varphi \rrbracket$ and we use $\mathbb{A}$ as a subscript of a component when it is not clear from the context, e.g., $\mathbb{I}_{-} \mathbb{\|}_{\mathbb{A}}: \Psi_{\mathbb{A}} \rightarrow 2^{\mathfrak{D}_{\mathbb{A}}}$.

\subsection{Words and Regexes}

The basic building blocks of regexes are predicates from an effective Boolean algebra CharClass of character classes, such as the class of digits, written as $\backslash \mathrm{d}$. Let $\mathfrak{D}=\mathfrak{D}_{\text {CharClass. }}$ A word over $\mathfrak{D}$ is a sequence of symbols $a_{1} \cdots a_{n} \in \mathfrak{D}^{*}$ and a language $\mathcal{L}$ over $\mathfrak{D}$ is a subset of $\mathfrak{D}^{*}$. We use $\epsilon$ to denote the empty word. The concatenation of words $u$ and $v$ is denoted as $u \cdot v$ (often abbreviated to $u v$ ) and is lifted to sets as usual. We call $a \in \mathfrak{D}$ the head of the word $a . w$ and $w \in \mathfrak{D}^{*}$ its tail. Furthermore, we write $\mathcal{L}^{n}$ for the $n$-th power of $\mathcal{L} \subseteq \mathfrak{D}^{*}$ with $\mathcal{L}^{0} \stackrel{\text { def }}{=}\{\epsilon\}$ and $\mathcal{L}^{n+1} \stackrel{\text { def }}{=} \mathcal{L}^{n} \cdot \mathcal{L}$.

The syntax of regexes is the following, with $\alpha \in \Psi_{\text {CharClass }}$ and $n, m \in \mathbb{N}, 0 \leq n, 0<m, n \leq m$ :

$$
\varepsilon \quad \alpha \quad R_{1} \cdot R_{2} \quad R_{1} \mid R_{2} \quad R\{n, m\} \quad R *
$$

where $R_{1} \cdot R_{2}$ is called a concat node and $R_{1} \mid R_{2}$ is called a choice node. The semantics of a regex $R$ is defined as a subset of $\mathfrak{D}^{*}$ in the following way: $\mathcal{L}(\alpha) \stackrel{\text { def }}{=} \llbracket \alpha \rrbracket, \mathcal{L}(\varepsilon) \stackrel{\text { def }}{=}\{\epsilon\}, \mathcal{L}\left(R_{1} R_{2}\right) \stackrel{\text { def }}{=} \mathcal{L}\left(R_{1}\right) \cdot \mathcal{L}\left(R_{2}\right)$, $\mathcal{L}\left(R_{1} \mid R_{2}\right) \stackrel{\text { def }}{=} \mathcal{L}\left(R_{1}\right) \cup \mathcal{L}\left(R_{2}\right), \mathcal{L}(R\{n, m\}) \stackrel{\text { def }}{=} \bigcup_{i=n}^{m}(\mathcal{L}(R))^{i}$, and $\mathcal{L}(R *) \stackrel{\text { def }}{=} \mathcal{L}(R)^{*} . R$ is nullable if $\epsilon \in \mathcal{L}(R)$. We will also need to refer to the number of character-class leaf nodes of a regex $R$, denoted by $\sharp_{\Psi}(R)$ and defined as follows: $\sharp_{\Psi}(\varepsilon)=0, \sharp_{\Psi}(\alpha)=1, \sharp_{\Psi}\left(R_{1} \cdot R_{2}\right)=\sharp_{\Psi}\left(R_{1} \mid R_{2}\right)=\sharp_{\Psi}\left(R_{1}\right)+\#_{\Psi}\left(R_{2}\right)$, $\sharp_{\Psi}(R\{n, m\})=\sharp_{\Psi}(R *)=\sharp_{\Psi}(R)$.

\subsection{Minterms}

Let $\operatorname{Preds}(R)$ be the set of all predicates that occur in a regex $R$, and let $\operatorname{Minterms}(R)$ denote the set of minterms of Preds $(R)$. Intuitively, $\operatorname{Minterms}(R)$ is a set of non-overlapping predicates that can be treated as a concrete finite alphabet. Each minterm is essentially a region in the Venn diagram of the predicates in $R$ : it is a satisfiable conjunction $\bigwedge_{\psi \in \operatorname{Preds}(R)} \psi^{\prime}$ where $\psi^{\prime} \in\{\psi, \neg \psi\}$. For example, if $R=$ $[0-\mathrm{z}]\{4\}[0-8]\{5\}$, then $\operatorname{Preds}(R)=\{[0-8],[0-\mathrm{z}]\}$ and $\operatorname{Minterms}(R)=\left\{[0-8],[9-\mathrm{z}],\left[{ }^{\wedge} 0-\mathrm{z}\right]\right\}$. Formally, if $\alpha \in \operatorname{Minterms}(R)$, then $\operatorname{Sat}(\alpha)$ and $\forall \psi \in \operatorname{Preds}(R): \llbracket \alpha \rrbracket \cap \llbracket \psi \rrbracket \neq \emptyset \Rightarrow \llbracket \alpha \rrbracket \subseteq \llbracket \psi \rrbracket$.

Note that although the number of minterms of a general set $X$ of predicates may be exponential in $|X|$, it is only linear if $X$ consists of intervals of symbols used in regexes, such as [a-zA-Z] or $[\wedge a-z A-Z]$ (the former denotes two intervals while the latter their complement, which is equivalent to the union of three intervals). Intervals of numbers generate only a linear number of minterms. 


\subsection{Symbolic Automata}

We use symbolic finite automata (FAs), whose alphabet is given by an effective Boolean algebra, as a generalization of classical finite automata. Formally, an FA is a tuple $A=\left(\mathbb{I}, Q, q_{0}, F, \Delta\right)$ where $\mathbb{I}$ is an effective Boolean algebra called the input algebra, $Q$ is a finite set of states, $q_{0} \in Q$ is the initial state, $F \subseteq Q$ is the set of final states, and $\Delta \subseteq Q \times \Psi_{\mathbb{I}} \times Q$ is a finite set of transitions. A transition $(q, \alpha, r) \in \Delta$ will be also written as $q-(\alpha) \rightarrow r$.

A run of $A$ from a state $p_{0}$ over a word $a_{1} \cdots a_{n}$ is a sequence of transitions $\left(p_{i-1}-\left(\alpha_{i}\right) \rightarrow p_{i}\right)_{i=1}^{n}$ with $a_{i} \in \llbracket \alpha_{i} \rrbracket$; the run is accepting if $p_{n} \in F$. The language of a state $q$, denoted $\mathcal{L}_{A}(q)$, is the set of words over which $A$ has an accepting run from $q$. The language of $A$, denoted $\mathcal{L}(A)$, is $\mathcal{L}_{A}\left(q_{0}\right)$. A classical finite automaton can be understood as an FA where the basic predicates have singleton set semantics, i.e., when for each concrete letter $a$ there is a predicate $\alpha_{a}$ such that $\llbracket \alpha_{a} \rrbracket=\{a\}$. A is deterministic iff for all $p \in Q$ and all transitions $p-(\alpha) \rightarrow q$ and $p-\left(\alpha^{\prime}\right) \rightarrow r$, it holds that if $\alpha \wedge \alpha^{\prime}$ is satisfiable, then $q=r$.

\section{COUNTING AUTOMATA}

Counting automata (CAs) are a natural and compact automata counterpart for regexes with counting. They are essentially a limited sub-class of classical counter automata, in which counters are only supposed to count the number of passes through some of its parts (corresponding to a counted sub-expression of a regex) and guards on transitions enforce a specified number of repetitions of that part before the automaton is allowed to move on.

Counter algebra. A counter algebra is an effective Boolean algebra $\mathbb{C}$ associated with a finite set $C$ of counters. The counters play the role of bounded loop variables associated with a lower bound $\boldsymbol{m i n}_{c} \geq 0$ and an upper bound $\boldsymbol{m a x}_{c}>0$ such that $\boldsymbol{m i n}_{c} \leq \boldsymbol{m a x}_{c} . \mathbb{D}_{\mathbb{C}}$ is the set of interpretations $\mathrm{m}: C \rightarrow \mathbb{N}$, called counter memories such that $0 \leq \mathfrak{m}(c) \leq \boldsymbol{m a x}_{c}$ for all $c \in C$. $\Psi_{\mathbb{C}}$ contains Boolean combinations of basic predicates CANEXIT ${ }_{c}$ and $\mathrm{CANINCR}_{c}$, for $c \in C$, whose semantics is given by

$$
\mathrm{m}=\operatorname{CANEXIT}_{c} \Longleftrightarrow \mathrm{m}(c) \geq \boldsymbol{m i n}_{c}, \quad \mathrm{~m} \mid=\mathrm{CANINCR}_{c} \Longleftrightarrow \mathrm{m}(c)<\boldsymbol{m a x}_{c}
$$

Counting automata. A counting automaton (CA) is a tuple $A=\left(\mathbb{I}, C, Q, q_{0}, F, \Delta\right)$ where $\mathbb{I}$ is an effective Boolean algebra called the input algebra, $C$ is a finite set of counters with an associated counter algebra $\mathbb{C}, Q$ is a finite set of states, $q_{0} \in Q$ is the initial state, $F: Q \rightarrow \Psi_{\mathbb{C}}$ is the final-state condition, and $\Delta \subseteq Q \times \Psi_{\mathbb{I}} \times(C \rightarrow O) \times Q$ is the (finite) transition relation, where $O=\{$ EXIT, INCR, EXIT1, NOOP $\}$ is the set of counter operations. The component $f$ of a transition $p-(\alpha, f) \rightarrow q \in \Delta$ is its (counter) operator. We often view $f$ as a set of indexed operations op $_{c}$ to denote the operation assigned to the counter $c, f(c)=$ op.

Semantics of CAs. The semantics of the CA $A$ is defined through its configuration automaton $F A(A)$, an FA whose states are $A$ 's configurations, i.e., pairs $(q, \mathfrak{m}) \in Q \times \mathfrak{D}_{\mathbb{C}}$ consisting of a state $q$ and a counter memory $m$. To define

$$
\begin{aligned}
& \operatorname{grd}\left(\mathrm{NOOP}_{c}\right) \stackrel{\text { def }}{=} \mathrm{T}_{\mathbb{C}} \\
& \operatorname{grd}\left(\mathrm{INCR}_{c}\right) \stackrel{\text { def }}{=} \mathrm{CANINCR}_{c} \quad u p d(\mathrm{INCR}) \stackrel{\text { def }}{=} \lambda n . n+1 \\
& \operatorname{grd}\left(\operatorname{EXIT}_{c}\right) \stackrel{\text { def }}{=} \mathrm{CANEXIT}_{c} \quad u p d(\mathrm{EXIT}) \stackrel{\text { def }}{=} \lambda n .0 \\
& \operatorname{grd}\left(\operatorname{ExIT}_{c}\right) \stackrel{\text { def }}{=} \operatorname{CANExIT}_{c} \quad u p d(\operatorname{EXIT} 1) \stackrel{\text { def }}{=} \lambda n .1
\end{aligned}
$$

$F A(A)$, we must first define the semantics of counter operators $f$, which occur on transitions. For this, we associate with each (indexed) operation $\mathrm{OP}_{c}$ a counter guard $\operatorname{grd}\left(\mathrm{OP}_{c}\right)$ and a counter update $u p d(\mathrm{OP})$ as shown on the right. Intuitively, the operation NOOP does not modify the counter's value and is always enabled. The operation INCR increments the counter and is enabled if the counter has not yet reached its upper bound. The operation ExIT resets the counter to 0 on exit from the counting loop and is enabled when the counter reaches its lower bound. The operation ExIT1 executes EXIT followed by INCR. The guard of a counter operator $f: C \rightarrow O$ is then a predicate $\varphi_{f} \in \Psi_{\mathbb{C}}$ over 
counter memories, and its update $\mathrm{f}: \mathfrak{D}_{\mathbb{C}} \cup\{\perp\} \rightarrow \mathfrak{D}_{\mathbb{C}} \cup\{\perp\}$ is a counter-memory transformer:

$$
\varphi_{f} \stackrel{\text { def }}{=} \bigwedge_{\mathrm{OP}_{c} \in f} \operatorname{grd}\left(\mathrm{OP}_{c}\right) \quad \mathrm{f}(\mathrm{m}) \stackrel{\text { def }}{=} \begin{cases}\lambda c \cdot u p d(f(c))(\mathrm{m}(c)) & \text { if } \mathrm{m}=\varphi_{f} \\ \perp & \text { otherwise }\end{cases}
$$

Intuitively, $\mathrm{f}$ updates all counters in a counter memory $m$ by their corresponding operations if $m$ satisfies the guard, otherwise the result is $\perp$.

We now define the configuration automaton of $A$, denoted as $F A(A)$, which defines the language semantics of the CA $A$. The states of $F A(A)$ are the configurations of $A$ (there are finitely many of them), and the initial state of $F A(A)$ is the initial configuration $\left(q_{0},\{c \mapsto 0 \mid c \in C\}\right)$ of $A$. A state $(p, \mathfrak{m})$ of $F A(A)$ is final iff $\mathfrak{m}=F(p)$. The transition relation $\Delta_{F A(A)}$ of $F A(A)$ is defined as

$$
\Delta_{F A(A)}=\left\{(p, \mathfrak{m})-(\alpha) \rightarrow(q, \mathrm{f}(\mathfrak{m}))|p-(\alpha, f) \rightarrow q \in \Delta, \mathfrak{m}|=\varphi_{f}\right\} .
$$

Deterministic and simple CAs. A is deterministic iff the following holds for every state $p \in Q$ and every two transitions $p-\left(\alpha_{1}, f_{1}\right) \rightarrow q_{1}, p-\left(\alpha_{2}, f_{2}\right) \rightarrow q_{2} \in \Delta$ : if both $\alpha_{1} \wedge \alpha_{2}$ and $\varphi_{f_{1}} \wedge \varphi_{f_{2}}$ are satisfiable, then $f_{1}=f_{2}$ and $q_{1}=q_{2}$. It follows from the definitions that, if $A$ is deterministic, then $F A(A)$ is deterministic too. $A$ is simple if for any two transitions $q-(\alpha, f) \rightarrow r$ and $q^{\prime}-\left(\alpha^{\prime}, f^{\prime}\right) \rightarrow r^{\prime}$, either $\alpha=\alpha^{\prime}$ or $\llbracket \alpha \rrbracket \cap \llbracket \alpha^{\prime} \rrbracket=\emptyset$. That is, different character guards do not overlap and can be mostly treated as plain symbols. We also require that all guards are satisfiable. CAs constructed from regexes by the algorithm in Section 5 will be simple.

Example 4.1. Fig. 1a shows a CA in an intuitive notation, with the initial state $q$ and final conditions $F(q)=\perp, F(s)=$ ExIT $_{c}$, where $\boldsymbol{m i n}_{c}=\boldsymbol{m a x}_{c}=100$. The same notation is used in Fig. 2 . Fig. 3a shows a CA in a notation following the formal development more closely.

\section{FROM A REGEX TO A CA VIA CONDITIONAL PARTIAL DERIVATIVES}

We introduce a generalization of the Antimirov's partial derivative construction [Antimirov 1996] to symbolic counting, which allows one to replace a verbose NFA by a succinct CA. The difference between the older variant of [Antimirov 1996] with explicit counting [Saarikivi et al. 2019] and our new version was already illustrated in Section 2 . To recall it briefly using the example of the regex $\cdot\{100\}$ : from 100 partial derivatives $\partial \cdot(\cdot\{i\})=.\{i-1\}, 1 \leq i \leq 100$, and an NFA with 100 states and transitions $.\{i\}-(\bullet) \cdot \bullet\{-1\}$, the new construction will take us to the single derivative $\partial_{.}(.\{100\})=\{.\{100\}\}$ associated with a conditional counter update which induce an NFA with a single state and the transition $.\{100\}-\left(\alpha, \mathrm{INCR}_{c}\right) \rightarrow \cdot\{100\}$.

We apply the construction on regexes that are normalized using the below rules where $X \leadsto Y$ denotes that $X$ is rewritten to $Y$ :

- All nested concat nodes are rewritten to the flattened right-associative list form, which is always maintained throughout the construction, using the rules: $(X \cdot Y) \cdot Z \leadsto X \cdot(Y \cdot Z)$, $\varepsilon \cdot Z \leadsto Z$, and $Z \cdot \varepsilon \leadsto Z$.

- If $S$ is nullable, then $S\{\ell, k\} \sim S\{0, k\}$. Moreover, in the nullable context $S\{0, k\}, S$ can be considered as if it was not nullable.

Observe that the normalization does not increase the size of the regex (it may decrease the size).

Let $R$ be a fixed normalized regex. A sub-expression of $R$ that is of the form $X=S\{\ell, k\}$ is called a counting loop. We consider each counting loop to represent a counter whose name is the counting loop itself and whose upper bound is $\boldsymbol{m a x}_{X}=k$ and lower bound is $\boldsymbol{m i n}_{X}=\ell$. For example, $(.\{9\}) *$ contains the counter $X=.\{9\}$ with $\boldsymbol{m i n}_{X}=\boldsymbol{m a x}_{X}=9$. In the following, let $C$ stand for the set of all counters that occur in $R$, also denoted by $\operatorname{Counters}(R)$.

We use the convention that the juxtaposition $X Y$ of normalized regexes $X$ and $Y$ is again a normalized regex of the equivalent concat node $X \cdot Y$ : e.g., if $X=a \cdot b$ and $Y=(a \cdot b) *$, then 
$X Y=a \cdot(b \cdot(a \cdot b) *)$. Observe in particular that $X \varepsilon=X$. In other words, we treat concatenated elements as sequences, and a singleton sequence equals to the element itself.

Our construction will work over the set $\Sigma=\operatorname{Minterms}(R)$ of minterms of $R$ and produce simple CA that use minterms of $\Sigma$ on transitions.

\subsection{Parametric Languages}

We define the language of a normalized regex starting with a counting loop relative to a counter value. For that, we lift the definition of languages to be parametric in counter memories $\mathrm{m}$, but regexes other than the above are treated as before with the memory $m$ passed through unchanged.

Recall that if $f$ is a counter operator and $m$ a counter memory, then $f(m)$ de-

$$
\begin{aligned}
& \mathrm{L}^{\mathrm{m}}(\varepsilon) \stackrel{\text { def }}{=}\{\epsilon\} \\
& \mathrm{L}^{\mathrm{m}}(\psi Z) \stackrel{\text { def }}{=} \llbracket \psi \rrbracket \cdot \mathrm{L}^{\mathrm{m}}(Z) \\
& \mathrm{L}^{\mathrm{m}}((W \mid Y) Z) \stackrel{\text { def }}{=} \mathrm{L}^{\mathrm{m}}(W Z) \cup \mathrm{L}^{\mathrm{m}}(Y Z) \\
& \mathrm{L}^{\mathrm{m}}(S * Z) \stackrel{\text { def }}{=} \mathrm{L}^{\mathrm{m}}(S)^{*} \cdot \mathrm{L}^{\mathrm{m}}(Z) \\
& \mathrm{L}^{\mathrm{m}}(S\{\ell, k\} Z) \stackrel{\text { def }}{=} \mathrm{L}^{\mathrm{m}}(S) \cdot \mathrm{L}^{\mathrm{INCR}_{S}\{\ell, k\}}(\mathrm{m})(S\{\ell, k\} Z) \cup \\
& \mathrm{L}^{\operatorname{EXIT}_{S}\{\ell, k\}(\mathrm{m})}(Z) \\
& \mathrm{L}^{\perp}(X) \stackrel{\text { def }}{=} \emptyset \quad(\text { for all } X)
\end{aligned}
$$

notes the appropriately updated memory where $\mathrm{f}(\mathrm{m})=\perp$ when $f$ is not enabled in $\mathrm{m}$. Below, if there is a single counter $c \in C$ such that $f(c) \neq$ NOOP, we sometimes identify $f$ with OP $_{c}$ and use $\mathrm{OP}_{c}(\mathrm{~m})$ to represent the updated memory $\mathrm{f}(\mathrm{m})$. Specifically, $\mathrm{INCR}_{X}$ (if enabled) increments the counter value of $X$ by 1 , and $\operatorname{ExIT}_{X}$ (if enabled) resets the counter value of $X$ to 0 . Let $m$ be a counter memory. Then Cases (1)-(6) define the parametric languages of regexes. The intuition behind Case (4) is that all counters that may be present in $S$ are inactive on the level of $S *$. Note also that Case (5) is well-defined since, for $X=S\{\ell, k\}$ and $\mathfrak{m}^{\prime}=\operatorname{INCR}_{X}(\mathfrak{m}), k-\mathfrak{m}^{\prime}(X)<k-\mathfrak{m}(X)$ if $\mathfrak{m}(X)<k$, and $\mathfrak{m}^{\prime}=\perp$ if $\mathfrak{m}(X)=k$.

Let $0 \stackrel{\text { def }}{=} \lambda c .0$ denote the initial memory that maps all counters to 0 . The below theorem, proven in [Turoňová et al. 2020], relates $L^{\mathrm{m}}(R)$ with the non-parametric definition of regular languages.

THeOREM 5.1. Let $R$ be a normalized regex. Then $\mathrm{L}^{0}(R)=\mathcal{L}(R)$.

\subsection{Conditional Derivation}

We will now introduce our conditional derivative construction formally.

A partial conditional derivative is a pair $\langle f, X\rangle$ where $f$ is a counter operator and $X$ a normalized regex. Given a counter memory $\mathrm{m}$, we let $\langle f, X\rangle$ define the language $\mathrm{L}^{\mathrm{m}}(\langle f, X\rangle) \stackrel{\text { def }}{=} \mathrm{L}^{\mathrm{f}(\mathrm{m})}(X)$. In other words, $f$ is first applied to the counter memory $m$ and then the regex is evaluated in the updated memory. If $f$ is not enabled in $\mathrm{m}$, then the denoted language is empty.

A conditional derivative is a finite set of partial conditional derivatives. The language defined by a conditional derivative $D$ in a counter memory $m$ is defined as the union of the languages of the partial conditional derivatives in $D: \mathrm{L}^{\mathfrak{m}}(D) \stackrel{\text { def }}{=} \bigcup_{d \in D} \mathrm{~L}^{\mathfrak{m}}(d)$.

To define how conditional derivatives of a given regex looks like, we need a notion of a sequential composition of conditional derivatives $D \otimes E \stackrel{\text { def }}{=}\{\langle f ; g, X \cdot Y\rangle \mid\langle f, X\rangle \in D,\langle g, Y\rangle \in E, f ; g \neq \perp\}$ where $f ; g \neq \perp$ is the composed counter operator such that $f ; g(m)=g(f(m))$. The case when $f ; g=\perp$ is discussed later on.

Conditional derivatives of a normalized regex are defined as shown on the right assuming that concatenations $X \cdot Y$ are normalized to the list form explained above, $\alpha \in \sum$, ID denotes the identity function $\lambda x . x$, and $X=S\{\ell, k\}$ is a counting loop. Observe that, in $\partial_{\alpha}(S) \otimes\left\{\left\langle\operatorname{INCR}_{X}, X Z\right\rangle\right\}$, the operation $\mathrm{INCR}_{X}$ gets composed with $\operatorname{NOOP}_{X}$, yielding $\operatorname{INCR}_{X}$ again, because $S\{\ell, k\}$ cannot occur in $S$. It is possible that in $\left\{\left\langle\operatorname{EXIT}_{X}, \varepsilon\right\rangle\right\} \otimes \partial_{\alpha}(Z), X$ is in scope of $Z$ (e.g., $Z$ starts with $X$ ). The composition can then contain the operation $\operatorname{EXIT}_{X} ; \operatorname{INCR}_{X}$ that corresponds to $\operatorname{EXIT} 1_{X}$ because $\operatorname{INCR}_{X}$ is trivially 
enabled when the counter value of $X$ is 0 . The only other possible composition of individual operations that can appear in this case is $\operatorname{EXIT}_{X} ; \operatorname{EXIT}_{X}$. If $\min _{X}=0, \operatorname{EXIT}_{X} ; \operatorname{EXIT}_{X}=\operatorname{EXIT}_{X}$, which is well-defined because $\operatorname{EXIT}_{X}$ is always enabled for $\boldsymbol{m i n}_{X}=0$. If $\boldsymbol{m i n}_{X}>$ 0 , then $\operatorname{EXIT}_{X} ; \operatorname{EXIT}_{X}$ is undefined, and $\operatorname{EXIT}_{X} ; \operatorname{EXIT}_{X}$ does not contribute anything to the composition. However, this is

$$
\begin{array}{rll}
\partial_{\alpha}(\varepsilon) & \stackrel{\text { def }}{=} & \emptyset \\
\partial_{\alpha}(\psi Z) & \stackrel{\text { def }}{=} \begin{cases}\{\langle\mathbf{I D}, Z\rangle\} & \text { if } \alpha \wedge \psi \text { is satisfiable } \\
\emptyset & \text { otherwise }\end{cases} \\
\partial_{\alpha}((W \mid Y) Z) \stackrel{\text { def }}{=} & \partial_{\alpha}(W Z) \cup \partial_{\alpha}(Y Z) \\
\partial_{\alpha}(S * Z) \stackrel{\text { def }}{=} & \partial_{\alpha}(S) \otimes\{\langle\mathbf{I D}, S * Z\rangle\} \cup \partial_{\alpha}(Z) \\
\partial_{\alpha}(X Z) \stackrel{\text { def }}{=} & \partial_{\alpha}(S) \otimes\left\{\left\langle\operatorname{INCR}_{X}, X Z\right\rangle\right\} \cup \\
& \left\{\left\langle\operatorname{EXIT}_{X}, \varepsilon\right\rangle\right\} \otimes \partial_{\alpha}(Z)
\end{array}
$$

correct since $X$ is not nullable, and the second $\operatorname{ExIT}_{X}$ is not enabled after the counter value of $X$ is reset to 0 . Intuitively, the second occurrence of $X$ cannot be exited without iterating $X$ at least once.

Example 5.2. Consider the regex $R=. \star \star a\{1,3\} a\{1,3\}$ a. Let $X$ be the counting loop a $\{1,3\} . R$ has two minterms a and [^a]. We get the following conditional derivatives of $R$, starting with the case for $\partial_{\alpha}(S * Z)$ due to the normal form assumption:

$$
\begin{aligned}
\partial_{\mathrm{a}}(R) & =\partial_{\mathrm{a}}(\bullet) \otimes\{\langle\mathbf{I D}, R\rangle\} \cup \partial_{\mathrm{a}}(X X \mathrm{a}) \\
& =\left\{\langle\mathbf{I D}, R\rangle,\left\langle\operatorname{INCR}_{X}, X X \mathrm{a}\right\rangle,\left\langle\operatorname{EXIT}_{X}, X \mathrm{a}\right\rangle\right\} \\
\partial_{\mathrm{a}}(X X \mathrm{a}) & =\partial_{\mathrm{a}}(\mathrm{a}) \otimes\left\{\left\langle\operatorname{INCR}_{X}, X X \mathrm{a}\right\rangle\right\}\left\{\left\langle\operatorname{EXIT}_{X}, \varepsilon\right\rangle\right\} \otimes \partial_{\mathrm{a}}(X \mathrm{a}) \\
& =\left\{\left\langle\operatorname{INCR}_{X}, X X \mathrm{a}\right\rangle\right\}\left\{\left\langle\operatorname{EXIT}_{X}, \varepsilon\right\rangle\right\} \otimes\left\{\left\langle\operatorname{INCR}_{X}, X \mathrm{a}\right\rangle,\left\langle\operatorname{EXIT}_{X}, \varepsilon\right\rangle\right\} \\
& =\left\{\left\langle\operatorname{INCR}_{X}, X X \mathrm{a}\right\rangle,\left\langle\operatorname{EXIT}_{X}, X \mathrm{a}\right\rangle\right\} \\
\partial_{\mathrm{a}}(X \mathrm{a}) & =\partial_{\mathrm{a}}(\mathrm{a}) \otimes\left\{\left\langle\operatorname{INCR}_{X}, X \mathrm{a}\right\rangle\right\} \cup\left\{\left\langle\operatorname{EXIT}_{X}, \varepsilon\right\rangle\right\} \otimes \partial_{\mathrm{a}}(\mathrm{a}) \\
& =\left\{\left\langle\operatorname{INCR}_{X}, X \mathrm{a}\right\rangle,\left\langle\operatorname{EXIT}_{X}, \varepsilon\right\rangle\right\} \\
\partial_{\mathrm{a}}(\mathrm{a})=\partial_{\mathrm{a}}(\bullet)=\partial_{[\wedge a]}(\cdot) & =\{\langle\mathbf{I D}, \varepsilon\rangle\} \\
\partial_{\left[{ }^{\wedge} \mathrm{a}\right]}(a) & =\emptyset
\end{aligned}
$$

Above, the composition $\operatorname{EXIT}_{X} ; \operatorname{EXIT}_{X}$ in $\partial_{a}(X X a)$ is undefined and thus removed. We also get that $\partial_{\left[{ }^{\wedge} a\right]}(R)=\{\langle\mathbf{I D}, R\rangle\}$ where $\partial_{\left[{ }^{\wedge} a\right]}(a)=\emptyset$ and consequently $\partial_{\left[{ }^{\wedge} a\right]}(X X a)=\emptyset$ and $\partial_{\left[{ }^{\wedge} a\right]}(X a)=\emptyset$.

If we now consider, for example, the language defined by $\partial_{a}(X a)$ in a valid counter memory $m$, it is the union of the languages $\mathrm{L}^{\mathrm{INCR}_{X}(\mathrm{~m})}(X \mathrm{a})$ and $\mathrm{L}^{\mathrm{EXIT}_{X}(\mathrm{~m})}(\varepsilon)$. These correspond to the cases of continuing to iterate the loop $X$ (if the counter value of $X$ is below 3 ) or exiting the loop (if the counter value of $X$ is at least 1$)$ and accepting $\{\epsilon\}$.

Example 5.3. Consider the regex $(.\{9\}) *$, whose CA is in Fig. 2. Here, . is the only input predicate and denotes the set of all characters. We explain the use of some of the counter operations in the CA of Fig. 2 by showing how they arise through the partial-derivative-based construction of CAs as discussed above. The initial state is the regex itself. The (only) partial derivative of the state $(.\{9\}) *$ is.$\{9\}(\cdot\{9\}) *$ where the body of the counting loop is exited but also incremented once, so EXIT1 is applied to $c$ under the guard CANExIT $_{c}$ (which is shown as $c \geq 9 / c:=1$ in the figure). The state $\cdot\{9\}(\cdot\{9\}) *$ has two cases of partial derivatives both leading back to $\cdot\{9\}(\cdot\{9\}) *$.

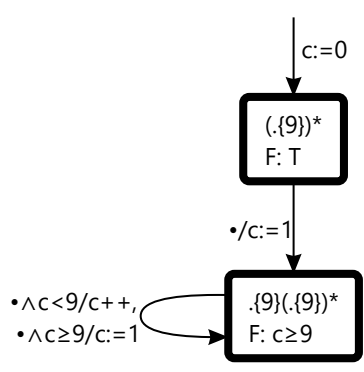

Fig. 2. $\mathrm{CA}((\cdot\{9\}) *)$

The first case is when $c<9$ (CANINCR $c$ holds), in which case $c$ is incremented (shown as $c<9 / c++$ in the figure). The second case is when the counting loop is conditionally nullable and is exited under the condition $\operatorname{canExIT}_{c}$ (i.e. $c \geq 9$ ), the value of $c$ is reset to 0 , and then $c$ is 
incremented as a result of taking the partial derivative of $(.\{9\}) *$. Thus, ExIT1 arises as a sequential composition of exiting the loop, followed by resetting the counter to 0 , and then incrementing it. Therefore, CANEXIT $_{c}$ must hold, while the increment condition holds trivially after a reset to 0 . The initial state is unconditionally final in Fig. 2, while the other state is final only when CANExIT $_{c}$ holds as marked by " $F$ :".

We now state the correctness theorem of conditional derivatives. For that, we define CANExit $R$ as the predicate shown above for a normalized regex $R$, assuming that $X$ stands for a counting loop.

$$
\operatorname{CANExiT}_{R} \stackrel{\text { def }}{=} \begin{cases}\mathrm{T}_{\mathbb{C}} & \text { if } R=\varepsilon, \\ \operatorname{CANExIT}_{Z} & \text { else if } R=Y Z \text { and } Y \text { is nullable, } \\ \operatorname{CANExIT}_{X} \wedge \operatorname{CANExIT}_{Z} & \text { else if } R=X Z, \\ \perp_{\mathbb{C}} & \text { otherwise. }\end{cases}
$$

Note that $Y$ above may also be a counting loop. However, since it is nullable, $\boldsymbol{m i n}_{Y}$ must be 0 , and then CANEXIT $_{Y}$ is always true. (If $\boldsymbol{m i n}_{Y}>0$, then $Y$ cannot be nullable as $R$ is normalized.)

We further need the following additional notions too. A counter $X$ is visible in $R$ if either $R=Y Z$ and $X=Y$, or else if $X$ does not occur in $Y$ and $X$ is visible in $Z$. A counter memory $m$ is valid for $R$ if $\mathfrak{m}(X)=0$ for all invisible counters $X$ that occur in $R$. Correctness of the construction of conditional derivatives is stated in Theorem 5.4-see [Turoňová et al. 2020] for a detailed proof.

THEOREM 5.4. Let $R$ be a normalized regex and let $\Sigma=\operatorname{Minterms}(\Theta)$ where $\Theta$ is some finite superset of Preds $(R)$. If $\mathrm{m}$ is valid for $R$, then $\mathrm{L}^{\mathfrak{m}}(R)=\bigcup_{\alpha \in \Sigma} \llbracket \alpha \rrbracket \cdot \mathrm{L}^{\mathfrak{m}}\left(\partial_{\alpha}(R)\right) \cup\left\{\epsilon|\mathfrak{m}|=\right.$ CANEXIT $\left._{R}\right\}$.

\subsection{Constructing CAs from Conditional Derivatives}

We convert a normalized regex $R$ to the counting automaton $\mathrm{CA}(R)$ whose set of states is the smallest set containing $R$ as the initial state and all those regexes that arise in conditional derivatives constructed from $R$ by repeated derivation wrt $\Sigma$. Given a state represented by a regex $S$, for each $\alpha \in \Sigma$ and each partial conditional derivative $\langle f, T\rangle \in \partial_{\alpha}(S)$, there is a transition $S-(\alpha, f) \rightarrow T$ in $\mathrm{CA}(R)$. The final condition $F(S)$ of a state $S$ of $\mathrm{CA}(R)$ is CANExiT $_{S}$. Observe that $F(S)=\perp_{\mathbb{C}}$ when $S$ is not nullable and has no visible counters, which corresponds to the classical case.

As shown in [Turoňová et al. 2020] the following result can be proved using Theorem 5.4.

Theorem 5.5. Let $R$ be a normalized regex and $A=F A(C A(R))$. Then, for all $\langle\mathrm{m}, S\rangle \in Q_{A}$, $\mathcal{L}_{A}(\langle\mathrm{~m}, S\rangle)=\mathrm{L}^{\mathrm{m}}(S)$.

The construction of $\mathrm{CA}(R)$ terminates, and the number of states of $\mathrm{CA}(R)$ is linear in $\sharp_{\Psi}(R)$.

TheOREM 5.6. Let $R$ be a normalized regex. Then $\left|Q_{C A(R)}\right| \leq \#_{\Psi}(R)+1$.

A proof of Theorem 5.6 is in [Turoňová et al. 2020]. We get the following final correctness result as a corollary of Theorem 5.5, Theorem 5.1, and Theorem 5.6.

COROLlary 5.7. Let $R$ be a normalized regex. Then $\mathcal{L}(R)=\mathcal{L}(C A(R))$.

Proof. First, $Q_{\mathrm{CA}(R)}$ is finite, and thus well-defined by using Theorem 5.6. Use Theorem 5.5 with $\langle\mathrm{m}, S\rangle$ as the initial state $\langle 0, R\rangle$ of $A$. It follows that $\mathcal{L}(A)=\mathrm{L}^{0}(R)$. Then use Theorem 5.1 for $\mathrm{L}^{0}(R)=\mathcal{L}(R)$ and $\mathcal{L}(\mathrm{CA}(R))=\mathcal{L}(A)$ holds by definition.

A further important aspect of $\mathrm{CA}(R)$ is that, although the number of input minterms may potentially be exponential in the number of predicates in $R$, in the case of predicates being represented as a finite union of intervals (as is done typically for character classes), the size of a single predicate representation can be estimated to be proportional to the number of interval borders in the union. In this case, the total size of all the minterms remains linear in the total size of all the predicates 
because the total number of interval borders will remain the same in minterms as in the original set of predicates. In other words, mintermization based on character classes does not blow up the number of transition in $\mathrm{CA}(R)$. We have also validated this fact experimentally.

\section{FROM COUNTING AUTOMATA TO COUNTING-SET AUTOMATA}

CAs obtained through conditional derivatives as shown in the previous section are nondeterministic. As one of the main contributions of this work, we now propose an approach for determinizing them into a form that can be used efficiently for regex matching. The approach from which we start and to which we contrast our new method is the naive determinization of CAs to DFAs: The given CA is first converted to its underlying NFA, by making the counter memories an explicit part of control states. The NFA is in turn determinized by the textbook subset construction.

Already the first step, the construction of the NFA, oftentimes explodes since it sacrifices the succinctness of symbolic counters (it is linear to the counter bounds). This initial blow-up is then much amplified in the subset construction, which is exponential to the size of the NFA and hence also to the counter bounds (as, e.g., in the case of the regex $* * a .\{k\}$ with its CA in Fig. 1a).

Our answer to this problem is a direct determinization of the CA into a novel type of automata, which we call counting-set automata ( $C s A s$ ). Control states of counting-set automata produced by our determinization are essentially the states of the corresponding DFA but with the counter memories removed. In order to be able to simulate a run of the DFA, they are equipped with special registers that can hold sets of integers, and they use them to compute the right counter memories at runtime. This completely avoids the state space explosion of the naive construction caused by wiring counter memories into control states. Moreover, the simulation is fast because all the manipulations with a counting set can be done in constant time.

\subsection{Counting-Set Automata}

We now formalize the idea of counting-set automata outlined above. We use the notion of a combined Boolean algebra $\mathbb{I} \times \mathbb{S}$, which allows us to manipulate pairs of predicates from the input algebra $\mathbb{I}$ and the counting-set algebra $\mathbb{S}$. For the purposes of this paper, we assume that predicates in $\Psi_{\mathbb{I} \times \mathbb{S}}$ have the form $\alpha \wedge \beta$ where $\alpha \in \Psi_{\mathbb{I}}$ and $\beta \in \Psi_{\mathbb{S}}$. The conjuction $(\alpha \wedge \beta) \wedge_{\mathbb{I} \times \mathbb{S}}\left(\alpha^{\prime} \wedge \beta^{\prime}\right)$ has the usual meaning of $\left(\alpha \wedge_{\mathbb{I}} \alpha^{\prime}\right) \wedge\left(\beta \wedge_{\mathbb{S}} \beta^{\prime}\right)$ and $\alpha \wedge \beta$ is satisfiable if both $\alpha$ and $\beta$ are satisfiable in their respective algebras.

Counting sets. We consider a set-based interpretation of counters where the value of a counter $c$ is a finite set rather than a single value. A counter under such an interpretation is referred to as a counting set. A (counting-)set memory for $C$ is a function $\mathfrak{s}: C \rightarrow \mathcal{P}_{\text {fin }}(\mathbb{N})$ such that, for all $c \in C$, $\operatorname{Max}(\mathfrak{s}(c)) \leq \max _{c} .{ }^{3}$ Observe that the set of all set memories for $C$ is finite. Counting-set predicates over $C$ form an effective Boolean algebra $\mathbb{S}_{C}$ called the counting-set algebra over $C$, also denoted just $\mathbb{S}$ when $C$ is clear from the context, whose domain $\mathbb{D}_{\mathbb{S}}$ is the set of all set memories for $C$. The set of predicates $\Psi_{\mathbb{S}}$ is the Boolean closure of the basic predicates CANINCR ${ }_{c}$ and $\mathrm{CANExIT}_{c}$, hence syntactically the same as in the counter algebra $\mathbb{C}$, but with a different semantics under $\mathbb{S}$ :

$$
\mathfrak{s}=\operatorname{CANExIT}_{c} \Longleftrightarrow \operatorname{Max}(\mathfrak{s}(c)) \geq \boldsymbol{m i n}_{c} \quad \text { and } \mathfrak{s}=\operatorname{CANINCR}_{c} \Longleftrightarrow \operatorname{Min}(\mathfrak{s}(c))<\max _{c}
$$

where $\operatorname{Min}(\cdot)$ and $\operatorname{Max}(\cdot)$ are the set minimum and maximum, respectively. Intuitively, the conditions test existence of a set element satisfying the same counter condition.

Counting-set automata. A counting-set automaton (CsA) is a tuple $A=\left(\mathbb{I}, C, Q, q_{0}, F, \Delta\right)$ where: $\mathbb{I}$ is an effective Boolean algebra called the input algebra. $C$ is a finite set of counters associated with the counting-set algebra $\mathbb{S}$. $Q$ is a finite set of states with $q_{0} \in Q$ being the initial state.

\footnotetext{
${ }^{3}$ We write $\mathcal{P}_{\text {fin }}(X)$ for the powerset of $X$ restricted to finite nonempty sets.
} 
$F: Q \rightarrow \Psi_{\mathbb{S}}$ is the final-state condition. $\Delta \subseteq Q \times \Psi_{\mathbb{I} \times \mathbb{S}} \times(C \rightarrow \mathcal{P}(O)) \times Q$ is a finite set of transitions. The second component is its guard. The third component is the counting-set operator in which $O=\{\mathrm{INCR}$, NoOp, Rst, Rst1 $\}$ is the set of counting-set operations. They are essentially counter operations lifted to sets (note the use of the larger initial letters to distinguish them from the counter operations). We also use the different names Rst and RsT1 for the lifting of EXIT and ExIT1 to stress their different usage (not only for exiting a loop but also for initialisation when entering the loop as will become clear in Eq. (7)). Sets of counting-set operations assigned to every counter by the counting-set operator are called combined (counting-set) operations.

The CsA $A$ is deterministic iff the following holds for every two transitions $p-\left(\psi_{1}, f_{1}\right) \rightarrow q_{1}$ and $p-\left(\psi_{2}, f_{2}\right) \rightarrow q_{2}$ in $\Delta$ : if $\psi_{1} \wedge \psi_{2}$ is satisfiable, then $f_{1}=f_{2}$ and $q_{1}=q_{2}$.

Semantics of CsAs. The semantics of an indexed counting-set operation $\mathrm{OP}_{c} \in O$ is the set transformer $u p d\left(\mathrm{OP}_{c}\right)$ defined as follows:

$$
\begin{aligned}
u p d\left(\mathrm{INCR}_{c}\right) & =\lambda S .\left\{n+1 \mid n \in S \wedge n<\max _{c}\right\} & \operatorname{upd}\left(\mathrm{RsT}_{c}\right) & =\lambda S .\{0\} \\
u p d\left(\mathrm{NoOP}_{c}\right) & =\lambda S . S & \operatorname{upd}\left(\mathrm{RsT}_{c}\right) & =\lambda S .\{1\}
\end{aligned}
$$

Then, the counting-set operator $f: C \rightarrow \mathcal{P}(O)$ is assigned the counting-set-memory transformer $f: \mathfrak{D}_{\mathbb{S}} \rightarrow \mathfrak{D}_{\mathbb{S}}$ defined as follows:

$$
\mathrm{f}(\mathfrak{s}) \stackrel{\text { def }}{=} \lambda c . \begin{cases}\bigcup_{\mathrm{op} \in f(c)} u p d\left(\mathrm{OP}_{c}\right)(\mathfrak{s}(c)) & \text { if } f(c) \neq \emptyset \\ \{0\} & \text { if } f(c)=\emptyset\end{cases}
$$

That is, (1) if $f(c) \neq \emptyset$, then the value $\mathfrak{s}(c)$ of each counting set $c$ is transformed into the union of the counting sets that result from applying the operations from $f(c)$ on $\mathfrak{s}(c)$, and (2) if $f(c)=\emptyset$, then $c$ is implicitly reset to $\{0\}$ (an implicit Rst). Our determinization procedure creates such transitions when the value of $c$ is irrelevant (when $c$ is a dead variable).

Note that, unlike counter operators of a CA, a counting-set operator $f$ does not induce any guard. The guard is rather a separate component of the transition. This is because CsA transitions produced in the CA-to-CsA determinization need guards that are partially independent of the operations of $f$. In particular, we will we need to distinguish cases such as $\neg \mathrm{CANExIT}_{c} \wedge \mathrm{CANINCR}_{c}$, $\mathrm{CANEXIT}_{c} \wedge \neg \mathrm{CANINCR}_{c}$, or CANEXIT ${ }_{c} \wedge \mathrm{CANINCR}_{c}$. The guard hence cannot be induced by $f$ alone

Note also that, unlike in CAs, the updates are defined for indexed operations. The reason is that the semantics of the INCR operation is restricted to never produce values greater than $\max _{c}$.

Finally, the language of the $C s A A$ is defined through its underlying configuration $F A, F A(A)$, as $\mathcal{L}(A):=\mathcal{L}(F A(A))$. The states of $F A(A)$ are configurations of $A$, namely, tuples of the form $(q, \mathfrak{s}) \in Q \times \mathfrak{D}_{\mathbb{S}}$ consisting of a state $q$ and a counting-set memory $\mathfrak{s}$. There are finitely many such configurations. The initial state of $F A(A)$ is the initial configuration $\left(q_{0},\{c \mapsto\{0\}\}_{c \in C}\right)$ of $A$ A transition $\tau=p-(\alpha \wedge \beta, f) \rightarrow q \in \Delta$ is enabled in a configuration $(p, \mathfrak{s})$ iff $\alpha$ is satisfiable and $\mathfrak{s} \in \llbracket \beta \rrbracket_{\mathbb{S}}$, meaning that $\mathfrak{s}$ satisfies the counter guard $\beta$. If $\tau$ is enabled in $(p, \mathfrak{s})$, then $F A(A)$ contains the transition $(p, \mathfrak{s})-(\alpha) \rightarrow(q, f(\mathfrak{s}))$. Finally, a state $(q, \mathfrak{s})$ of $F A(A)$ is final iff $\mathfrak{s}=F(q)$.

Example 6.1. An example of a CsA is in Fig. 1b. It uses intuitive notations that were also introduced in Section 2 as abbreviations for the operations of the counting-set data structure. Counting-set operators are depicted as assignments to $c$, Rst is represented as $\{0\}$ on the right of the assignment, Rst 1 is represented by $\{1\}$, INCR by $c+1$, and Noop by $c$. Multiple transitions between the same states and with the same updates are merged into one with a simplified guard. An example whose notation closely follows the formal development is in Fig. 3.

Runtime efficiency of counting sets. A major reason for choosing CsAs as the target kind of machine for determinization of CAs is that pattern matching with CsAs is fast. Using the data structure explained in Section 2, all the basic counting-set tests and updates, namely, CANINCR $_{c}$, 
CANEXIT $_{c}$, Noop, INCR, Rst, and Rst1, can be implemented to run in constant time regardless of the size of the counting set and the value $\boldsymbol{m a x}_{c}$ (assuming constant-time complexity of integer arithmetic operations). Moreover, almost all combined counting-set operations can be implemented to run in constant time too. In particular, when at most one counting-set operation of a given combined operation returns a set other than $\{0\}$ or $\{1\}$, their union can be computed in constant time. However, the union of two general sets, other than $\{0\}$ and $\{1\}$, would take time linear to the size of the sets (which is at most $\boldsymbol{m a x}_{\boldsymbol{c}}$ ). The only operations that may return sets other than $\{0\}$ or $\{1\}$ are NoOP and INCR. We therefore call a transition slow if its counting-set operator $f$ assigns to some counter $c$ the result of a combined operation $f(c)$ that contains both NoOP and INCR. A CsA that has slow transitions is called slow, and a CsA that does not have them is called fast. Slow CsAs are fortunately rare in practice (cf. Section 7).

When a fast CsA is used in pattern matching, tests and updates of one counting set then take $O(1)$ time, which in turn gives $O(|C|)$ for all counting sets and their unions. This is our major achievement: the independence of the running time from the counter bounds.

\subsection{Encoding DFA Powerstates as CsA Configurations}

In order to build intuition needed for understanding our determinization algorithm, we will first concretize how the configurations of a CsA can encode states of a DFA corresponding to the NFA $F A(A)$ underlying a given $\mathrm{CA} A=\left(\mathbb{I}, C, Q, q_{0}, F, \Delta\right)$. First, recall that, since $A$ is converted into $F A(A)$ by making the counter memories explicit parts of control states, the states of $F A(A)$ are pairs $(p, \mathfrak{m})$ consisting of a state $p$ of $A$ and a counter memory $m$. Second, assume that $F A(A)$ is determinized using the textbook subset construction. ${ }^{4}$ We denote the result as $D F A(A)$ from now on. Then, the states of $D F A(A)$ are sets of states of $F A(A)$, i.e., sets of pairs $(p, \mathfrak{m})$, which we will call powerstates The control states of the CsA $A^{\prime}$ built by our CA-to-CsA determinization will be subsets of the set $Q$ of states of the CA $A$. The configurations of $A^{\prime}$ will thus be pairs $(R, \mathfrak{s})$ where $R \subseteq Q$ is a CsA control state, i.e., a set of states of $A$, and $\mathfrak{s}: C \rightarrow \mathcal{P}_{\text {fin }}(\mathbb{N})$ is a counting-set memory. Let us now consider how $\mathfrak{s}$ can be interpreted in this context.

Naive encoding. A naive interpretation of a CsA configuration $(R, \mathfrak{s})$ is a DFA state containing all pairs $(r, \mathfrak{m})$ such that $r \in R$ and, for all $c \in C, \mathfrak{m}(c)$ can be any value from $\mathfrak{s}(c)$. The set of the counter memories $m$ is then isomorphic to the Cartesian product $\prod_{c \in C} \mathfrak{s}(c)$ of the sets $\mathfrak{s}(c)$ assigned to the counters, and the entire powerstate is the Cartesian product $R \times \mathrm{m}$ of the set of states and the set of counter memories. The naive interpretation, however, is too impractical as it cannot express any dependence of a counter memory on the CA state (every state can be paired with each considered memory) nor any mutual dependence of values of different counters within a counter memory (every possible value of a counter $c$ can be paired with every possible value of any other counter d). Most DFAs compiled from real-life regexes do not fit into this representation. For instance, the DFA configuration $\{(q, c=0),(s, c=0),(s, c=1)\}$ of the CA from Fig. 1 in Section 2 could not be represented by a CsA configuration because $q$ and $s$ appear with different sets of counter values.

Encoding with counter scopes. Our key observation how to resolve the above problem (at least for many real-life scenarios) is to take advantage of that not every counter is "used" at every CA state. In fact, the value of a counter is usually implicitly 0 at most states except a few. If these states are known, the implicit zeros do not have to be remembered explicitly in the counting sets, and the encoding becomes much more flexible. To formalize this, we introduce the notion of the scope of a

\footnotetext{
${ }^{4}$ The DFA produced by the textbook subset construction from a simple FA $\mathcal{A}=\left(\mathbb{I}, Q, q_{0}, F, \Delta\right)$ will have $\mathcal{P}(Q)$ as the set of states, transitions $S-(\alpha) \rightarrow\{r \in Q \mid s-(\alpha) \rightarrow r \in \Delta, s \in S\}$, the initial state $\left\{q_{0}\right\}$, and as the final states all those intersecting $F$. We note that to determinize a CA which is not simple, one could start from the more sophisticated version of the subset construction for symbolic automata of [Veanes et al. 2010], which avoids explicit generation of all minterms.
} 
counter that over-approximates the set of states where a counter $c$ can have a non-zero value and that is easy to compute. ${ }^{5}$ The scope is defined inductively as the smallest set of states $\sigma(c)$ such that

(1) $q \in \sigma(c)$ if there is a transition to $q$ with either $\mathrm{INCR}_{c}$ or $\operatorname{EXIT} 1_{c}$, or

(2) there is a transition to $q$ from a state in $\sigma(c)$ with the $\operatorname{NOOP}_{c}$ operation.

In other words, the scope of $c$ spreads from an increment of $c$ along the transition relation until a transition with $\operatorname{EXIT}_{c}$.

The DFA powerstate encoded by a CsA configuration $(R, \mathfrak{s})$ can then be formally defined as the set $(R, \mathfrak{s})^{D F A}$ of configurations $(r, \mathfrak{m})$ of the CA $A$ such that $r \in R$ and, for all $c \in C, \mathfrak{m}(c) \in \mathfrak{s}(c)$ if $c \in \sigma(r)$, else $\mathrm{m}(c)=0$. We call the powerstates of $D F A(A)$ that can be encoded by CsA configurations Cartesian, and call the entire DFA Cartesian if all its powerstates are Cartesian.

Example 6.2. The powerstates of the $D F A(A)$ of the CA $A$ from Fig. 1a are indeed Cartesian (as discussed in Section 2) because $q_{0}$ is not in the scope of $c$. The encoding of powerstates by CsA configurations is also illustrated in Section 2 and later also in Example 6.4.

The Cartesian encoding still cannot express all kinds of DFA powerstates. In particular, it cannot express more subtle dependencies of counter values on the state, and dependencies of counter values of different counters on each other, which mainly concerns CAs with nested counting loops compiled from regexes with nested counting sub-expressions. Example 6.5 discusses a regex that leads to a non-Cartesian CA. However, we later present a strong empirical evidence that a significant majority of real-life regexes lead to Cartesian CA.

\subsection{Generalized Subset Construction}

We will now describe the core of our CA-to-CsA determinization. It is built on top of the textbook subset construction for NFAs. We use the CA from Fig. 3a as a running example through the section. We make a simplifying assumption that the input CAs are simple (different character classes on their transitions do not overlap). This is satisfied by CAs generated by the derivative construction from Section 5 since their transitions are labeled by minterms of the original regex. The assumption could be dropped and the construction could be relatively easily generalized in the style of symbolic automata determinization of [Veanes et al. 2010].

Let $A=\left(\mathbb{I}, C, Q, q_{0}, F, \Delta\right)$ be a simple CA with the scope function $\sigma: Q \rightarrow \mathcal{P}(C)$. The algorithm produces the deterministic $\operatorname{CsA} A^{\prime}=\left(\mathbb{I}, C, Q^{\prime}, S_{0}, F^{\prime}, \Delta^{\prime}\right)$ whose components are constructed as described below. Namely, control states of $A^{\prime}$, called powerstates, are subsets of $Q$, i.e., $Q^{\prime} \subseteq \mathcal{P}(Q)$. The initial powerstate is $S_{0}=\left\{q_{0}\right\}$. A powerstate $S \in Q^{\prime}$ is final iff the final condition holds for some of its elements, i.e., $F^{\prime}(S) \stackrel{\text { def }}{=} \bigvee_{q \in S} F(q)$. The sets $\Delta^{\prime}$ and $Q^{\prime}$ are constructed by a fixpoint computation that explores the state space reachable from $S_{0}$. During the construction, transitions starting from previously reached powerstates are constructed and included together with their target states into $\Delta^{\prime}$ and $Q^{\prime}$, respectively, until no new powerstates can be reached.

Transitions starting from a given control state $R$ of the CsA $A^{\prime}$ are constructed to update the runtime values of counting sets such that they simulate transitions of the DFA corresponding to the CA $A$. Assume a CsA configuration $(R, \mathfrak{s})$ and a DFA transition $(R, \mathfrak{s})^{D F A}-(\alpha) \rightarrow P$ from the DFA powerstate encoded by $(R, \mathfrak{s})$ over an input minterm $\alpha$. The simulating CsA transition must transform $(R, \mathfrak{s})$ into $\left(R^{\prime}, \mathfrak{s}^{\prime}\right)$ with $\left(R^{\prime}, \mathfrak{s}^{\prime}\right)^{D F A}=P$. The simulated DFA transition was constructed from $\alpha$-transitions of the NFA $F A(A)$ that are actually instantiations of the CA $\alpha$-transitions enabled

\footnotetext{
${ }^{5}$ Computing the precise set of states where a counter $c$ can have a non-zero value would require a reachability analysis in the general case (since some of the transitions may never be executable-think of simultaneously counting with counters $c$ and $d$ such that $\mathrm{CANINCR}_{c}<\mathrm{CANEXIT}_{d}$, then the exit transition for $d$ will never be taken). For CAs produced by our derivative construction, the scope, however, corresponds to this set precisely-no transitions that are never executable are generated.
} 
in configurations $(r, \mathfrak{m}) \in(R, \mathfrak{s})^{D F A}$. The simulating CsA transition will be constructed from these CA transitions. They can be identified by (1) their source state, which must be in $R,(2)$ an alphabet minterm $\alpha \in \sum$ where $\Sigma$ is the set of minterms over all input predicates in the CA $A$, and (3) their compatibility with a particular set of enabled/disabled counter guards. This set of guards belongs to the set of minterms $\Gamma_{R, \alpha}$ of the set of counter guards on the $\alpha$-transitions originating in $R$ :

$$
\Gamma_{R, \alpha} \stackrel{\text { def }}{=} \operatorname{Minterms}\left(\left\{\operatorname{grd}\left(\mathrm{OP}_{c}\right) \mid r-(\alpha, f) \rightarrow s \in \Delta, r \in R \wedge c \in \sigma(r), \mathrm{OP}_{c} \in f\right\}\right) .
$$

Hence, the CsA will have a transition leaving $R$ for each $\alpha \in \Sigma$ and $\beta \in \Gamma_{R, \alpha}$, and the transition will be built from the set of CA $\alpha$-transitions originating in $R$ and consistent with $\beta$ :

$$
\Delta_{R, \alpha, \beta} \stackrel{\text { def }}{=}\left\{r-(\alpha, f) \rightarrow s \in \Delta \mid r \in R, \boldsymbol{S a t}\left(\varphi_{f} \wedge \beta\right)\right\} .
$$

Its target is the set $T$ of all target states of the transitions in $\Delta_{R, \alpha, \beta}$, and its guard is $\alpha \wedge \beta$. $^{6}$

The remaining component is the counting-set operator $f^{\prime}$. It must summarize the updates of the counter values on transitions of $\Delta_{R, \alpha, \beta}$ as updates of the respective counting sets. The values of counters that are out of scope, hence implicitly zero, will not be tracked in counting sets. Tracking the value of a counter hence starts when $A^{\prime}$ simulates a transition of $A$ entering the scope of the counter, and ends when no state from the scope is present in the target CsA state.

Let $\Delta_{R, \alpha, \beta}(c)$ be the set of transitions in $\Delta_{R, \alpha, \beta}$ with the target state in the scope of $c$. The countingset operator $f^{\prime}$ is built in the form $f^{\prime}(c) \stackrel{\text { def }}{=}\{o p(\tau, c) \mid$ $\left.\tau \in \Delta_{R, \alpha, \beta}(c)\right\}$. Here, op $(\tau, c)$ denotes the countingset operation that, given a CA transition $\tau=\stackrel{\text { def }}{=}$ $p-(\alpha, f) \rightarrow q$, transforms the set of possible values of the counter $c$ at the state $p$ to the set of values obtained at $q$ after taking the transition. It is defined

$$
o p(p-(\alpha, f) \rightarrow q, c) \stackrel{\text { def }}{=}
$$

$= \begin{cases}\text { NoOP } & \text { if } f(c)=\text { NOOP } \wedge p \in \sigma(c) \\ \text { INCR } & \text { if } f(c)=\text { INCR } \wedge p \in \sigma(c) \\ \text { RsT } & \text { if } f(c)=\text { NOOP } \wedge p \notin \sigma(c) \\ \text { RsT1 } & \text { if } f(c)=\text { INCR } \wedge p \notin \sigma(c) \\ \text { RsT } & \text { if } f(c)=\text { EXIT } \\ \text { RsT1 } & \text { if } f(c)=\text { EXIT1 }\end{cases}$

in Eq. (7) on the right. The set operation induced by the CA transition corresponds to the counter operation on the transition. In the third and fourth case, when the CA transition comes from out of the scope, it is certain that the counter can only have the value 0 , which is the same value as produced by EXIT (or EXIT1 when the counter is immediately incremented). The resulting CsA transition is therefore $S-\left(\alpha \wedge \beta, f^{\prime}\right) \rightarrow T$. Note that $f^{\prime}(c)$ ends up empty when the target powerstate is fully out of the scope of $c$, which semantically corresponds to the implicit reset to $\{0\}$.

Observe that $A^{\prime}$ is deterministic since, for any two distinct transitions $S-(\alpha, f) \rightarrow T$ and $S-\left(\alpha^{\prime}, f^{\prime}\right) \rightarrow T^{\prime}$, the condition $\alpha \wedge \alpha^{\prime}$ is unsatisfiable by virtue of minterms.

Theorem 6.3. For the $C A A$ and the $C s A A^{\prime}$ above, we have $\mathcal{L}\left(A^{\prime}\right) \supseteq \mathcal{L}(A)$ and $\left|Q^{\prime}\right| \leq 2^{|Q|}$.

Proof (IDEA). The language inclusion is proved by showing that the configuration automaton $F A\left(A^{\prime}\right)$ of $A^{\prime}$ simulates $D F A(A)$, more concretely, that each configuration $(R, \mathfrak{s})$ of $A^{\prime}$, a state of $F A\left(A^{\prime}\right)$, simulates the powerstate $(R, \mathfrak{s})$ of $D F A(A)$. The bound on the size of the state space follows from that states of the CsA are sets of states of the CA.

Example 6.4. Consider the CA in Fig. 3 a that has states $q_{0}, q_{1}$, and $q_{2}$. The state $q_{0}$ is initial, the final condition of $q_{2}$ is $\mathrm{T}$, and it is $\perp$ for $q_{0}$ and $q_{1}$. The set of counters is $C=\{c\}$ with $\sigma(c)=\left\{q_{1}\right\}$ (i.e., $c$ is not used and hence implicitly 0 in $q_{0}$ and $q_{2}$ ). Finally, $\Sigma=\{a,[\wedge a]\}$. In Fig. 3a, we compactly represent transitions over all minterms from $\Sigma$ using .. The determinization starts exploring the CsA from its initial state $S_{0}=\left\{q_{0}\right\}$.

Let us focus on the transitions for the input minterm $\alpha=\mathrm{a}$. Two transitions are leaving $q_{0}$, namely $\delta_{1}=q_{0}-\left(a\right.$, NOOP $\left._{c}\right) \rightarrow q_{0}$ and $\delta_{2}=q_{0}-\left(a\right.$, NOOP $\left._{c}\right) \rightarrow q_{1}$, both with no guard on $c$, hence $\Gamma_{S_{0}, \alpha}=\{T\}$. The

\footnotetext{
${ }^{6}$ Recall that the predicates in $\Psi_{\mathbb{C}}$ and $\Psi_{\mathbb{S}}$ are syntactically the same.
} 


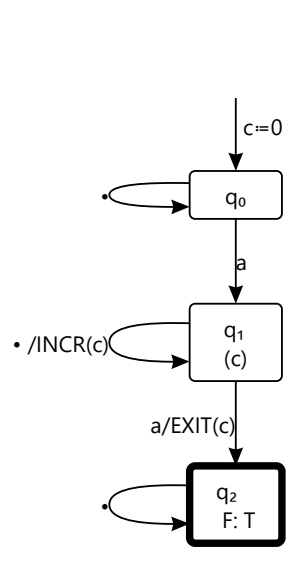

(a) The CA for $* * a \cdot\{4,8\}$ a

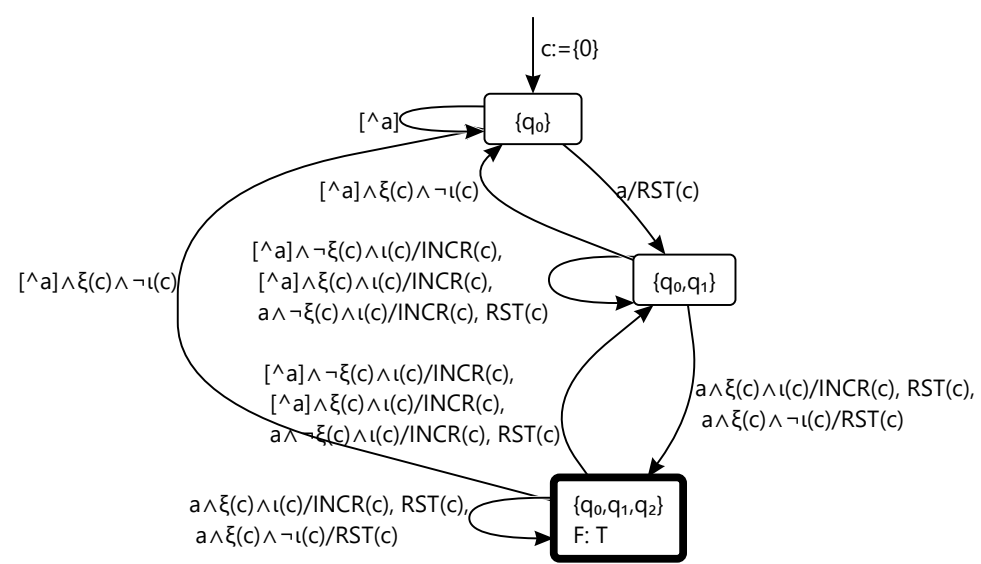

(b) Determinization of the CA into a CsA

Fig. 3. From a regex via a CA to a deterministic CsA. We are using a notation closely following the formal development. We only use OP(c) instead of $\mathrm{OP}_{c}$ and abbreviate CANEXIT $c$ by $\xi(c)$ and $\operatorname{CANINCR}_{c}$ by $l(c)$.

guard $\mathrm{T}$ is thus the only choice for the counter minterm $\beta$. The set $\Delta_{R, \alpha, \beta}$ of transitions consistent with $\alpha$ and $\beta$ then contains both a-transitions $\delta_{1}$ and $\delta_{2}$ originating from $q_{0}$. Since $\delta_{2}$ is entering the scope of $c$, it generates the counting-set operation $\operatorname{RsT}_{c}$ according to the third case of Eq. (7). Since $\delta_{1}$ stays out of the scope, it does not generate any counting-set operations. We obtain the counting-set operator $f^{\prime}=\left\{\operatorname{RsT}_{c}\right\}$ and generate the CsA transition $\tau_{1}=\left\{q_{0}\right\}-\left(\mathrm{a} \wedge \beta,\left\{\operatorname{RsT}_{c}\right\}\right) \rightarrow\left\{q_{0}, q_{1}\right\}$.

Next, let us focus on the a-transitions from $S_{1}=\left\{q_{0}, q_{1}\right\}$. Here, $\Gamma_{S_{1} \text {, a }}$ has the following three satisfiable elements: $\mathrm{CANEXIT}_{c} \wedge \mathrm{CANINCR}_{c}, \neg \mathrm{CANExIT}_{c} \wedge \mathrm{CANINCR}_{c}$, and $\mathrm{CANEXIT}_{c} \wedge \neg \mathrm{CANINCR}_{c}$ (the guard $\neg \mathrm{CANEXIT}_{c} \wedge \neg \mathrm{CANINCR}_{c}$ is excluded as it is never satisfied for non-empty sets of positive integers). Let us generate a transition for the second case, $\beta=\neg$ CANExIT $_{c} \wedge$ CANINCR $_{c}$. We obtain $\Delta_{S_{1}, \mathrm{a}, \beta}=\left\{q_{0}-\left(\mathrm{a}, \mathrm{NOOP}_{c}\right) \rightarrow q_{0}, q_{0}-\left(\mathrm{a}, \mathrm{NOOP}_{c}\right) \rightarrow q_{1}, q_{1}-\left(\mathrm{a}, \mathrm{INCR}_{c}\right) \rightarrow q_{1}\right\}$. As before, the first transition does not contribute to $f^{\prime}$ as it stays out of the scope, and the second transition adds Rst $\mathrm{R}_{c}$. The third transition adds $\mathrm{INCR}_{c}$ (the second case of Eq. (7)). The resulting CsA transition is thus $\tau_{2}=$ $S_{1}-\left(\mathrm{a} \wedge \neg \mathrm{CANEXIT}_{\mathcal{c}} \wedge \mathrm{CANINCR}_{\mathcal{c}},\left\{\operatorname{INCR}_{\mathcal{c}}, \mathrm{RsT}_{c}\right\}\right) \rightarrow S_{1}$. The rest of the construction is analogous.

Last, let us also illustrate the simulation of $D F A(A)$ by the constructed CsA transitions. On the word $a a$, the DFA would execute the run $\left\{\left(q_{0}, c=0\right)\right\}-(a) \rightarrow\left\{\left(q_{0}, c=0\right),\left(q_{1}, c=0\right)\right\}-(a) \rightarrow\left\{\left(q_{0}, c=\right.\right.$ $\left.0),\left(q_{1}, c=0\right),\left(q_{1}, c=1\right)\right\}$. The simulating run of our CsA would start in the initial configuration $\left\{\left\{q_{0}\right\}, c \in\{0\}\right\}$. The transition $\tau_{1}$ would produce the configuration $\left\{\left\{q_{0}, q_{1}\right\}, c \in\{0\}\right\}$ (since $\operatorname{RsT}(\{0\})=\{0\})$ from where $\tau_{2}$ would produce $\left\{\left\{q_{0}, q_{1}\right\}, c \in\{0,1\}\right\}$ (since $\operatorname{INCR}(\{0\})=\{1\}$ and $\operatorname{RsT}(\{0\})=\{0\})$. The sequence of configurations precisely encodes the sequence of the DFA powerstates, that is, the sequnce $\left(\left\{q_{0}\right\}, c \in\{0\}\right)^{D F A}=\left\{\left(q_{0}, c=0\right)\right\} ;\left(\left\{q_{0}, q_{1}\right\}, c \in\{0\}\right)^{D F A}=$ $\left\{\left(q_{0}, c=0\right),\left(q_{1}, c=0\right)\right\}$; and $\left(\left\{q_{0}, q_{1}\right\}, c \in\{0,1\}\right)^{D F A}=\left\{\left(q_{0}, c=0\right),\left(q_{1}, c=0\right),\left(q_{1}, c=1\right)\right\}$ (recall that $q_{0}$ is not in the scope of $c$, hence $c$ has implicitly the value 0 there).

\subsection{Uniformity: A Sufficient Semantic Correctness Criterion}

Given a CA $A$, we produce a CsA $A^{\prime}$ that may overapproximate $A$ in terms of the language. We explain how this may happen and present conditions under which the language stays unchanged. In particular, the overapproximation is caused by non-Cartesian powerstates of $D F A(A)$. (Recall that, in a Cartesian powerstate, states in the scope of a counter must appear with the same set of values of that counter.) A configuration of the CsA cannot encode a non-Cartesian powerstate precisely, it can only overapproximate it. A larger powerstate may then accept a larger language. 
Example 6.5. Take $R=(\mathrm{a} \mid \mathrm{aa})\{5\}$ and the $\mathrm{CA}(R)$ shown in Fig. 4. After reading the word $a a, D F A(\mathrm{CA}(R))$ reaches the powerstate $\left\{\left(q_{0}, c=1\right),\left(q_{0}, c=2\right),\left(q_{1}, c=2\right)\right\}$, which is not Cartesian because both states are in the scope of the counter $c$ but are paired with different counter values. Our CsA would reach the configuration $\left(\left\{q_{0}, q_{1}\right\}, c \in\{0,1,2\}\right)$, which encodes the larger powerstate $\left\{\left(q_{0}, c=0\right),\left(q_{0}, c=1\right),\left(q_{0}, c=2\right),\left(q_{1}, c=0\right),\left(q_{1}, c=1\right),\left(q_{1}, c=\right.\right.$ $2)\}$ where both states appear with both counter values.

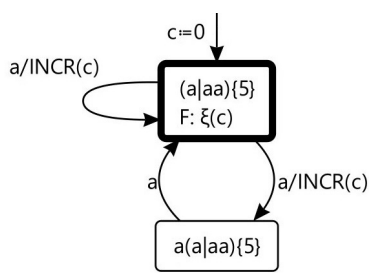

Fig. 4. $C A((\mathrm{a} \mid \mathrm{aa})\{5\})$.

Uniformity. We now introduce the so-called uniformity of a CA as a property under which determinization preserves the language. Uniformity prevents creation of non-Cartesian powerstates. It includes two conditions.

The first condition prevents the kind of scenario from Example 6.5. For each DFA transition $\tau^{\prime}$, it requires that every CA state $q$ that is in the scope of some counter $c$ within the DFA state to which $\tau^{\prime}$ leads receives the same set of values of $c$. This requires testing whether the sets of transitions covered by $\tau^{\prime}$ and incoming to every such CA state $q$ induce the same CsA operations for $c$.

The second condition prohibits two counters from being active at once, a scenario which arises from regexes with nested counting. Indeed, the relation between values of two simultaneously active counters may easily become more intricate than what can be expressed by a Cartesian product of two sets (consider, e.g., the regex $a$ ? $(a\{1\} a)\{2\}$ and the word $a a a)$. The condition requires testing that no state appears in the scope of two counters.

Formally, given a CsA transition $\tau^{\prime}=S-\left(\alpha \wedge \beta, f^{\prime}\right) \rightarrow T$, a counter $c$, and a CA state $q \in \sigma(c)$, we define the set $f_{q}^{\prime}(c)$ of incoming CsA operations for $c$ induced by the incoming transitions of $q$ from which $\tau^{\prime}$ is built ( $\alpha$-transitions consistent with $\beta$ originating in $S$ ) as follows:

$$
f_{q}^{\prime}(c) \stackrel{\text { def }}{=}\left\{o p(\tau, c) \mid \tau \in \Delta_{S, \alpha, \beta(c)} \wedge \text { the target of } \tau \text { is } q\right\} .
$$

We call the transition $\tau^{\prime}$ uniform iff, for each counter $c \in C$, any two states $q, r \in \sigma(c) \cap T$ have the same sets of incoming CsA operations, i.e., $f_{q}^{\prime}(c)=f_{r}^{\prime}(c)$. The CA $A$ is then uniform if all transitions of $A^{\prime}$ are uniform and if no state of $A$ appears in the scope of two counters.

Theorem 6.6. If a $C A A$ is uniform, then $\mathcal{L}(A)=\mathcal{L}\left(A^{\prime}\right)$.

Proof (IDEA). By showing bisimilarity between states $q$ of $F A\left(A^{\prime}\right)$, i.e., configurations of the CsA $A^{\prime}$ and powerstates $q^{D F A}$ of $D F A(A)$.

Uniformity can be checked on the fly, while constructing $A^{\prime}$. It is also automatically implied when the $\mathrm{CA}$ is constructed from certain classes of regexes, as discussed below.

\subsection{Syntactic Correctness Criteria}

Uniformity is only a semantic property. Below, we show examples of actual regexes that do and do not lead to uniform CAs and discuss some simple syntactic classes of regexes that imply uniformity. A detailed study of syntactic classes of regexes that guarantee uniformity is, however, beyond the scope of this paper and a part of our future work.

The regexes that induce non-uniform CAs are often those where, intuitively, there is a position in some input text that may either be matched against the first character of a counted sub-expression or against some inner character of the same sub-expression. In such a situation, there may be two runs of the induced CA: one that increments the associated counter (the increment happens) at that position and moves to some state $q$, and the other that leaves the counter as it is, while in its scope, and moves into a different state $r$. The counter value then depends on the state: it is different in $q$ and in $r$. The corresponding DFA state is then non-Cartesian and the CA is non-uniform. 
Example 6.7. We present several commented examples of regexes with non-uniform CAs where our determinization overapproximates the language of the obtained CsA.

- (a|ab|ba) $\{5\}$ - the string aba could be matched as "a" followed by "ba", having incremented the counter twice, or as "ab" that is followed by the prefix "a" of "ab", having incremented the counter once only.

- $a\{1,3\} a\{3\}$ - this case can be explained similarly as the previous one. Alternatively, note that, assuming that our translation to a CA produces two counters, say $c_{1}$ and $c_{2}$, then after reading $n$ letters $a$, the CA needs to remember that $c_{1}+c_{2}=n$. Such non-trivial relations between counter values are not Cartesian.

- .*(aa) $\{6\}$ - assuming a sequence of $a$ 's on the input, the counter may be either incremented on odd characters and left unchanged on even ones, or the other way around. As the counter values depend on the position within the "aa" (and hence on the CA state), the CA cannot be uniform. Note that the prefix $\bullet *$ is quite usual as it corresponds to searching for the regex (aa) $\{6\}$ anywhere in the input string.

- .*(a\{2\}) $\{2\}-$ after reading $a a$, if the value of the outer counter is 1 , then the value of the inner counter must be 0 . This is a non-trivial relation between the values of the two counters, which is not Cartesian. Nested counting is often problematic, however, many of such examples may still be solved quite efficiently by unfolding one of the counters.

Syntactic classes of regexes that guarantee uniformity. A simple class of regexes that guarantees uniformity is a generalization of the class of monadic regexes of [Holík et al. 2019] (where counting is allowed over character classes only). Namely, it consists of regexes with counting loops of the form

$$
\left(\alpha_{1} \ldots \alpha_{n}\right)\{\ell, k\} \text { s.t. } \llbracket \alpha_{1} \rrbracket \text { is disjoint from every } \llbracket \alpha_{i} \rrbracket, 1<i \leq n .
$$

Intuitively, the disjointness with $\alpha_{1}$ ensures that the generated CA will only be able to process $\alpha_{1}$ through an increment transition at the beginning of a new iteration of the loop, with no possibility of having a conflicting NOOP transition that could read the same symbol inside the body of the loop (which is exactly what happens with the second symbol $a$ in Example 6.5). The CsA compiled form this class are also guaranteed to be fast.

\section{EXPERIMENTAL EVALUATION}

We have implemented our approach in a C\# prototype called CA available at [Turoňová et al. [n.d.]] (see [Turoňová et al. 2020] for details how to efficiently implement CsAs) and evaluated its pattern matching capabilities against other state-of-the-art regex matchers on patterns that use the counting operator. We focused on comparison against Google's RE2 library [Google [n.d.]] $]^{7}$, an automata-based matcher designed to be fast, predictable, and resilient against ReDoS attacks. We also include other three efficient matchers into the comparison, namely the standard GNU grep program [Haertel et al. [n.d.]] (version 3.3), the .NET standard library regex matcher from System. Text. RegularExpressions [Microsoft 2020], and Symbolic Regex Matcher (SRM) [Saarikivi et al. 2019].

Let us shortly summarize how the tools work. The main algorithms of RE2 and grep implement optimized versions of the Thompson's on-the-fly determinization where the constructed DFA states are cached. The construction has a bound on the size of the DFA-if the bound is reached, the so-far constructed DFA states are flushed to avoid consuming too much memory. In some situations when caching is found ineffectual, RE2 turns the caching off, and the performance can drop even lower (see the description in [Cox 2010] for details). We note that RE2 rejects an input regex if it contains a counting operator with a bound bigger than 1,000. SRM is based on symbolic derivatives

\footnotetext{
${ }^{7}$ We used the version 2019-01-01 of RE2 via the command line interface re2g from https://github.com/akamai/re2g.
} 
constructed on the fly, also in the spirit of the Thompson's algorithm, and, likewise, bases its efficiency on caching (in fact, SRM is quite close to an implementation of the Thompson's algorithm over CAs with caching). The .NET matcher uses a backtracking algorithm over NFAs, while our CA eagerly constructs a deterministic CsA for the input regex. The former four are mature tools, and especially RE2 and grep contain many high- and low-level optimizations, such as using the Boyer-Moore algorithm [Boyer and Moore 1977] to skip over many characters that are known to not be a part of a match. RE2 and grep are compiled programs while CA, SRM, and .NET run within the .NET Framework (therefore, they have some inherent overhead due to the just-in-time compilation at start-up and its inability to use advanced code optimizations, as well as garbage collection). Note that even though the tools based on the on-the-fly subset construction (RE2, grep, and SRM) are linear to the lenght of the text, they still take space exponential to the counter bounds in the worst case, by creating sets of the size linear to the counter bounds, exponential to their decadic encoding used in the regex.

We run our benchmarks on a machine with the Intel(R) Xeon(R) CPU E3-1240 v3 @ 3.40 GHz running Debian GNU/Linux (we use the Mono platform [project [n.d.]] to run .NET tools). To avoid issues with generating exact matches, which might differ for different tools, the tools were run in the setting where they counted the number of lines matching ${ }^{8}$ the given regex (e.g. the $-c$ flag of grep).

\subsection{ReDoS Resiliency}

Our main experiment focuses on the resilience of the matching engines against ReDoS attacks. The regexes used for this experiment were selected (1) from the database of over 500,000 real-world regexes coming from an Internet-wide analysis of regexes collected from over 190,000 software projects [Davis et al. 2019]; (2) from databases of regexes used by network intrusion detection systems (NIDSes), in particular, Snort [M. Roesch et al. [n.d.]], Bro [Robin Sommer et al. [n.d.]], Sagan [The Sagan team [n.d.]], and the academic papers [Češka et al. 2018; Yang et al. 2010]; (4) the RegExLib database of regexes [RegExLib.com [n.d.]]; and (5) industrial regexes from [Holík et al. 2019], used for security purposes. From these, we created our set of benchmarks by the following steps:

(1) We selected regexes that contained counting loops whose sum of upper bounds was larger than 20. This let us focus on regexes where the use of counting makes sense (there are surprisingly many regexes occurring in practice where the use of a counting loop is unnecessary, e.g., regexes containing sub-expressions similar to $a\{0,1\}$ or even just $a\{1\})$. Moreover, we also removed all except 26 regexes with counters bigger than 1,000, which cannot be handled by RE2. We left the 26 regexes as representatives of "large" counters. This left us with 5,000 regexes.

(2) Then, we filtered out regexes $R$ such that either $\mathrm{CA}(R)$ was not uniform (i.e., the CsA produced by our algorithm was not precise, cf. Section 6.4), or such that the $C s A$ was not fast (i.e. not all counting-set operators were constant-time, cf. Section 6.1). After this step, a vast majority, 4,429 of the regexes, remained.

(3) For the regexes that remained, we used a lightweight ReDoS generator designed to exploit counting (cf. Section 7.3) to generate $\sim 10 \mathrm{MiB}$ long input texts. In particular, we managed to generate "adversarial" input texts for 1,789 regexes (for the rest of the regexes, either the underlying state space was too small, so the generator could not construct the text, or the generation hit the timeout of $600 \mathrm{~s}$ ). Our benchmark data set is available at [Holík et al. 2020].

We ran all tools on the generated benchmarks (counting the number of lines of the input text matching the regex) and give scatter plots comparing the running times of the tools in Fig. $5 \mathrm{a}$

\footnotetext{
${ }^{8}$ We consider the standard semantics of "matching" used by grep, i.e., a line matches a regex $R$ if it contains a string that is in $\mathcal{L}(R)$, unless it contains start-of-line $\left(^{\wedge}\right)$ or end-of-line $(\$)$ anchors, in which case the matched string needs to occur at the start and/or at the end of the line respectively.
} 


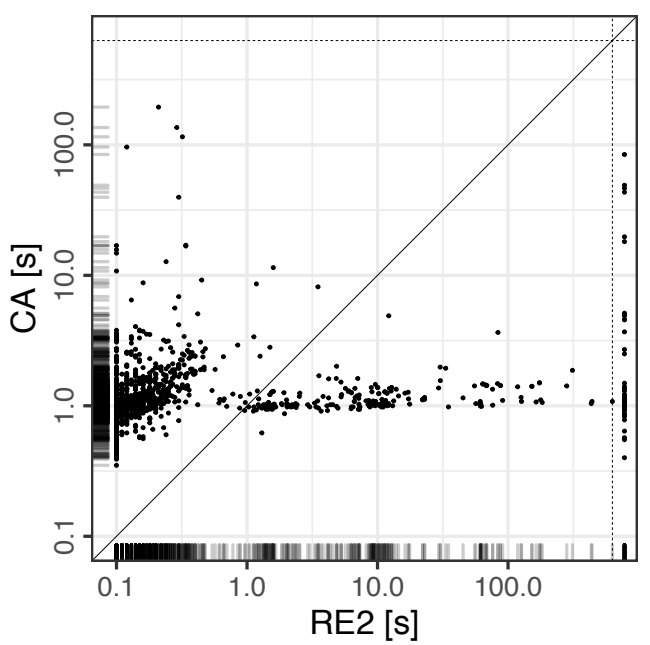

(a) The comparison of running times of CA and RE2 on our benchmark set (CA wins: 287/1,789)

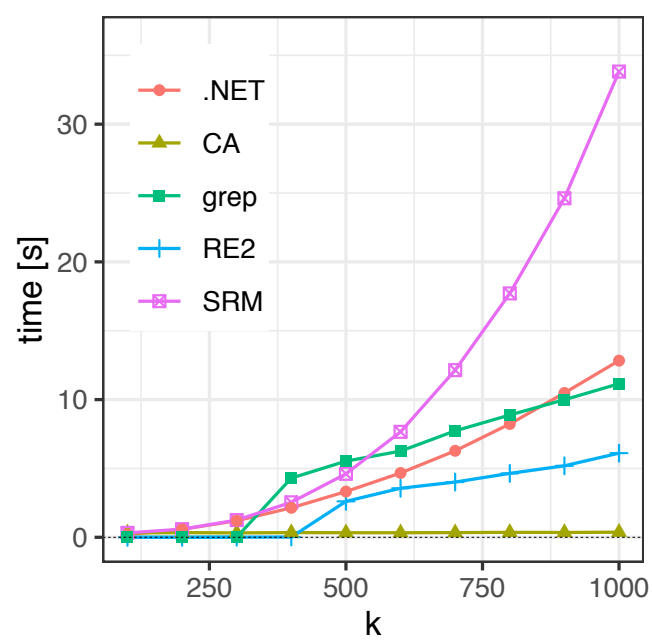

(b) Running times of the tools on the regex "(_a ) $\{k\}_{-} a$ " where $k$ is a parameter

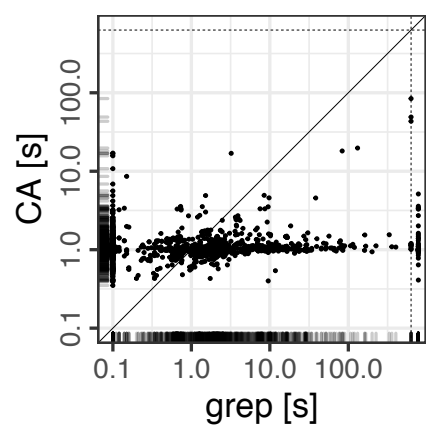

(CA wins: $862 / 1,425)$

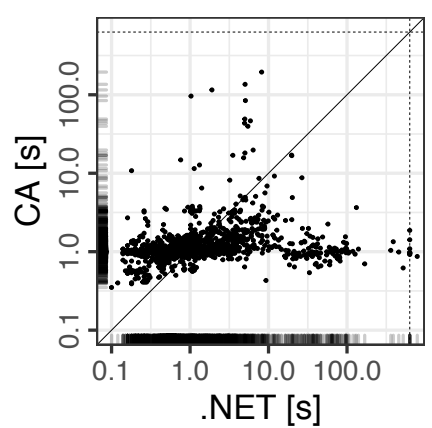

(CA wins: 708/1,789)

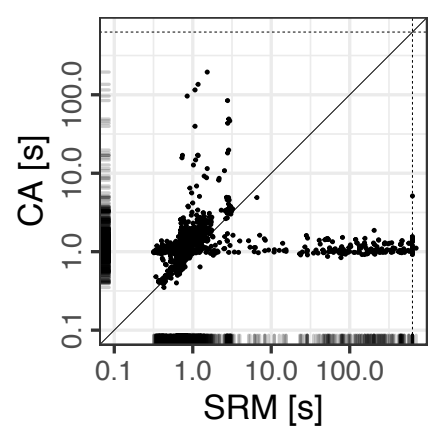

(CA wins: 345/1,789)

(c) The comparison of running times of CA with grep, .NET, and SRM on our benchmark set

Fig. 5. Graphs with results of our experiments. Note that, in (a) and (c), the axes are logarithmic, the dashed lines denote the timeout $(600 \mathrm{~s})$, and the data points between the dashed lines and the edge of a plot represent benchmarks where the tool did not run successfully. We also provide the number of times CA won.

and Fig. 5c (the timeout was $600 \mathrm{~s}$ ). On the bottom and the left-hand side of every plot, there are rug plots illustrating the distribution of the data points. Note that the axes are logarithmic, so the difference between data points grows as these points are away from zero (in particular, differences of values smaller than $1 \mathrm{~s}$ are negligible). The semantics of regexes supported by grep differs from the one supported by other tools, so we only considered the cases when the number of matches was the same when comparing with grep). In the plots, the data points between the dashed lines and edges of the plots represent errors, e.g. due to the regex being rejected (for counters $>1,000$ for RE2) or being interpreted using a different semantics (in the case of grep).

In Fig. 5a, we compare CA with RE2. We wish to point out the following interesting observations. Although RE2 wins more often on the whole benchmark set (our prototype does not include the many advanced optimizations present in RE2), there is a number of benchmarks (287) where its performance significantly deteriorates, and CA is faster. In particular, there are 89 benchmarks 
Table 1. Statistics for the graphs in Fig. 5 (times are given in seconds). For CA, we provide several times: "total" is the total time, "CA" is the time for translating a regex into a (nondeterministic) CA, "CsA" is the time of determinization of the CA into a CsA, and "match" is the time spent when matching the input text.

\begin{tabular}{lrrrrrrrr}
\hline & \multirow{2}{*}{ RE2 } & grep & .NET & \multicolumn{2}{c}{ SRM } & \multicolumn{4}{c}{ CA } \\
& & & & & total & CA & CsA & match \\
\hline mean & 36.11 & 34.38 & 9.12 & 26.78 & 1.73 & 0.05 & 0.23 & 0.69 \\
median & 0.10 & 0.70 & 0.76 & 0.73 & 1.03 & 0.03 & 0.04 & 0.68 \\
std. dev & 157.05 & 147.17 & 52.10 & 106.16 & 7.27 & 0.29 & 2.73 & 0.29 \\
timeouts & 1 & 11 & 8 & 16 & 0 & & & \\
\hline
\end{tabular}

where the time of RE2 is bigger than $10 \mathrm{~s}$, i.e., its speed drops below $1 \mathrm{MiB} / \mathrm{s}$ (we consider this speed of processing denotes a successful ReDoS attack, even though the limit may be significantly larger in practice ${ }^{9}$ ). For CA, the number of benchmarks that took over $10 \mathrm{~s}$ was only 22 ; in fact, all except 3 benchmarks finished within $100 \mathrm{~s}$-the blow-up in these 3 benchmarks is not caused by the counters but rather by many "|" and "?" operators, so over $70 \%$ of the total time is spent by constructing the CsA. If used, e.g., in an NIDS, the CsA would be created only once and then used for matching giga-/terabytes of data, so the initial overhead could be neglected.

Comparing with the other tools (Fig. 5c) and also clearly visible in the corresponding rug plots and the statistics in Table 1, we can observe that the performance of CA is much more robust than the performance of the other tools; the mean time and standard deviation of CA is significantly lower than the rest of the tools. In particular, from the benchmarks where CA was faster than RE2, the time of CA on all except two benchmarks was almost the same (including them, the standard deviation was 0.37 ). We provide four times for CA: "total": the total user time of matching (measured using the GNU time utility), "CA": the time for translating the input regex into a CA, "CsA": the time it took to determinize the CA into a CsA, and "match": the time of matching the input text with the CsA. Note that, in the tables, there is a noticeable discrepancy between the sum "CA" + "CsA" + "match" and "total", which is due to the .NET Framework overhead, such as just-in-time compilation and (in particular) the garbage collector.

In Table 2, we give a selection of interesting benchmarks. These contain benchmarks that are difficult for usually more than one tool. We emphasize the benchmarks coming from the NIDSes Snort and Bro. Notice that, for most of them, matching using RE2 (and also other tools) gets extremely slow. Slow matching over these regexes can have disastrous consequences for network security, potentially completely eliminating a given NIDS.

The CsAs produced by CA were also much smaller than the corresponding DFAs. The CsAs have on average 29 states (median: 7) and 306 transitions (median: 11). On the other hand, classical NFAs constructed from the regexes have on average 112 states (median: 52), and when determinized, the resulting DFAs have on average 2,802 states (median: 67) and 10,384 transitions (median: 107). Using CsAs significantly lowers the chance that determinization explodes.

7.1.1 The Effect of Nondeterministic Counting. We say that a regex contains nondeterministic counting if, when translated into a CA $A$ using the algorithm in Section 5, there is a word $w$ such that $A$ can over $w$ reach two configurations with different values of some counter.

Regexes with nondeterministic counting are the main focus of our benchmark. Namely, they constitute $67 \%$ of the 1,789 regexes used. From the 1,284 regexes that were at least slightly problematic for some of the other tools except CA (it took some tool $\geq 1 \mathrm{~s}$ ), $73 \%$ of them were with

\footnotetext{
${ }^{9}$ The required processing speed depends on the application. NIDSes performing deep packet inspection may require a line-processing speed of units or tens of GiB/s [Češka et al. 2018], while application servers validating user inputs may suffice with units or tens of $\mathrm{MiB} / \mathrm{s}$.
} 
Table 2. Selection of interesting benchmarks. "TO" denotes a timeout (600 s) and "-" denotes an error. Due to space constraints, in the "Regex" column, “..." denotes omitted parts of the regexes (we tried to preserve the parts containing occurrences of the repetition operator) and “ ” denotes breaking a regex into two lines. In the column source, Sw denotes the regexes collected in [Davis et al. 2019] from software projects.

\begin{tabular}{|c|c|c|c|c|c|c|c|c|c|}
\hline \multirow[t]{2}{*}{ Source } & \multirow[t]{2}{*}{ Regex } & \multirow[t]{2}{*}{ RE2 } & \multirow[t]{2}{*}{ grep } & \multirow[t]{2}{*}{.NET } & \multirow[t]{2}{*}{ SRM } & \multicolumn{4}{|c|}{ CA } \\
\hline & & & & & & total & CA & CsA & match \\
\hline Snort & $\begin{array}{l}. *[a A][\mathrm{uU}][\mathrm{tT}][\mathrm{hH}] \ldots[\mathrm{iI}][\mathrm{cC}] \sim \\
\sim[\wedge \backslash \mathrm{x} \oslash \mathrm{A}]\{512\}\end{array}$ & 11.27 & 7.8 & 361.1 & 555.56 & 1.04 & 0.03 & 0.05 & 0.31 \\
\hline Snort & $\backslash \times 20[\wedge \backslash \times 21 \backslash \times 22]\{500\}$ & 439.98 & 0.11 & 2.20 & TO & 1.08 & 0.03 & 0.04 & 0.83 \\
\hline Snort & \begin{tabular}{l}
${ }^{\wedge}$ RCPT TO\} \backslash x 2 0 \backslash s * [ \backslash w \backslash s @ \backslash . ] \{ 2 0 0 , \} \sim $\\
{\sim \backslash x 20[\backslash w \backslash s @ \backslash .]\{200,\} \ldots}$ & 340.7 & - & TO & TO & 1.68 & 0.03 & 0.07 & 0.89 \\
\hline Snort & php. $* \backslash \times 20[\wedge \backslash n]\{256\}$ & 176.75 & 0.10 & 1.22 & TO & 1.08 & 0.04 & 0.07 & 0.74 \\
\hline Snort & $\begin{array}{l}\wedge(N T \mid \text { CallBack } \mid \text { SID } \mid \text { TimeOut }) \backslash \mathrm{s} * \sim \\
\sim \backslash \mathrm{x} 20 \backslash \mathrm{s} *\left[{ }^{\wedge} \backslash \mathrm{n}\right]\{512\}\end{array}$ & 164.11 & 0.12 & 14.59 & 229.41 & 1.07 & 0.03 & 0.07 & 0.72 \\
\hline Snort &.$\star[n N][e E][w W] \ldots\left[^{\wedge} \backslash \times 20\right]\{100\}$ & 0.13 & 1.26 & 39.92 & 0.74 & 0.81 & 0.03 & 0.04 & 0.65 \\
\hline Bro & $\begin{array}{l}\wedge[\mathrm{nN}][\mathrm{aA}][\mathrm{mM}][\mathrm{eE}]=\mathrm{s} *[\wedge \backslash \mathrm{\wedge} \backslash \mathrm{n} \backslash \mathrm{x} 3 \mathrm{~b} \sim \\
\sim \backslash \mathrm{x} 20 \backslash \mathrm{x} 09 \backslash \mathrm{x} 0 \mathrm{~b} \backslash \mathrm{x} 2 \mathrm{c}]\{300\}\end{array}$ & 128.57 & 12.24 & 0.51 & 76.48 & 1.15 & 0.03 & 0.04 & 0.94 \\
\hline Sw & $-\cdot\{39\}$ & 22.96 & 225.34 & 1.94 & 357.68 & 1.12 & 0.03 & 0.04 & 0.79 \\
\hline Sw & $(.\{1,980\}[],) \backslash s+(\backslash S)$ & 260.59 & TO & 308.66 & 0.63 & 1.07 & 0.03 & 0.05 & 0.59 \\
\hline Sw & $\left(\_a\right)\{64999\} \_a$ & - & - & TO & TO & 0.96 & 0.03 & 0.04 & 0.51 \\
\hline Sw & $\backslash[\{50000\} a \backslash]\{50000\}$ & - & - & 4.36 & TO & 5.13 & 0.02 & 0.02 & 0.41 \\
\hline Sw & $\begin{array}{l}{ }^{\wedge} \mathrm{QS}([\mathrm{NDR}])(.\{4\})(.\{6\})(\backslash \mathrm{d}\{8\}) \ldots \sim \\
\sim(.\{4\})(.\{6\})(.\{8\})(.\{8\})(.) \$\end{array}$ & 0.12 & 0.10 & 1.03 & 0.85 & 96.20 & 0.04 & 81.64 & 0.65 \\
\hline
\end{tabular}
\end{tabular}

nondeterministic counting. From the 454 regexes that were significantly problematic for some of the other tools (it took some tool $\geq 10 \mathrm{~s}$ ), $85 \%$ of them had nondeterministic counting. From the 109 regexes that were problematic for all other tools ( $\geq 1 \mathrm{~s}), 100 \%$ were with nondeterministic counting. As shown in the results above, our approach can deal with nondeterministic counting quite well.

7.1.2 Adversarial Regexes. Another ReDoS scenario is when the attacker can control the regex to be used for matching. Creating a counting regex causing efficiency problems for a given text is easier than generating adversarial texts. For instance, the regex $[a-z A-Z() ., '] *[a-z A-Z]$ $[a-z A-Z() ; ']\{250\}$ was obtained as a modification of the running example ".*a. $\{k\}$ " (where a appears $k$ positions from the end). When run on a $\sim 4 \mathrm{MiB}$ English text with sufficiently long lines, RE2 took $86 \mathrm{~s}$, grep took $26 \mathrm{~s}$, while CA took only $1.1 \mathrm{~s}$. Similar examples could be obtained from regexes from Section 7.1 for which some specific difficult text can be generated, namely by widening their character classes.. Our approach solves a large class of the dangerous cases, allowing one to significantly alleviate restrictions put on the user for security/efficiency reasons.

\subsection{Robustness wrt Counter Values}

This experiment measures the ability of the tools to cope with increasing counter bounds. For this, we selected the regex " $\left(\_a\right)\{k\}_{-} a$ " where $k$ is a parameter (the original regex (_a ) $\{64999\}_{-} a$ comes from [Davis et al. 2019]) and measured the time the tools took on a $\sim 500 \mathrm{KiB}$ text created by our generator for increasing values of $k$. We give the results in Fig. $5 \mathrm{~b}$ (the timeout was $40 \mathrm{~s}$ ).

With the increasing value of $k$, the time needed by CA stays constant, around $0.35 \mathrm{~s}$, while the time needed by other tools grows. In particular, .NET and SRM have cubic trends wrt the value of $k$, while RE2 and grep grow linearly. Notice that, for RE2 and grep, their matching time is low (around $0.01 \mathrm{~s}$ ) until they reach a threshold from which they start behaving linearly. This corresponds to the situation when the size of the cache for storing states of the NFA-to-DFA construction is not enough to accommodate the DFA states exercised by the input adversarial text. This yields repeated flushing of the cache, making it ineffectual. 


\subsection{Adversarial Text Generation}

RE2 and grep store powerstates of the NFA-to-DFA construction in a cache. In typical cases, the amount of cache misses is low and almost the entire text is processed using the cache, which is extremely fast. If the cache, however, exceeds a given size, it is flushed. If the input text is such that the DFA run sees many different states, then cache misses are frequent, so large powerstates need to be constructed often, and the performance of the matching drops.

Therefore, we focus on generating texts that force exploration of many new large powerstates. In essence, we explore the configuration space of the CsA with the goal of finding as many large configurations as possible, with the focus on generating large counting sets. We partially drive the search towards loops in the CsA structure that have a potential to create large counting sets: the loops use counters with large bounds, do not contain exits, and contain RsT or RsT1 operations. For space reasons, we omit the technical details here; perfecting this method for stress testing automata-based matchers is, however, one of our future goals.

\subsection{A Note on the Maturity of the Tools}

The aim of our experiments is comparing algorithms rather than tools, and it should be noted that CA is much less optimized than the rest. This holds especially for RE2 and grep, which have both been actively developed for over 10 years and the amount of engineering effort invested into making them fast is substantial. The optimizations are both high-level, such as using the Boyer-Moore algorithm for skipping sections of the input text, and low-level, such as using $\mathrm{C} / \mathrm{C}++$, on-the-fly determinization, or optimizing memory accesses [Cox 2010; Haertel [n.d.]]. On the other hand, although there have been some optimizations done in CA (such as finding a start of a match), their nature is still quite simple. The three tools are, however, all based on the same principle of using deterministic automata, and many of the optimizations and heuristics in RE2 and grep (at least all of those mentioned above) could be directly re-applied in our setting. SRM builds on the .NET framework and reuses the .NET regex parser while replacing the built-in backtracking back-end matcher with a matching engine based on Brzozowski-style symbolic derivatives to create the DFA on the fly. In fact, CA builds on the open-source codebase of SRM and extends it with counters.

\section{RELATED WORK}

Regexes and their derivatives. Brzozowski derivatives [Brzozowski 1964] provide a practical approach to incrementally creating a DFA from a regex and can be used for efficient matching [Fischer et al. 2010; Owens et al. 2009] and match generation [Saarikivi et al. 2019]. Efficient determinization based on Brzozowski derivatives was first investigated in [Berry and Sethi 1986]. In the classical setting, Antimirov derivatives [Antimirov 1996] are used to construct NFAs from regexes, and may in some cases result in exponentially more succinct automata than the corresponding DFAs constructed with Brzozowski derivatives. The precise connection between conditional derivatives defined in Section 5 and Antimirov derivatives is that, without counting loops, $\left\{D \mid\langle\mathbf{I D}, D\rangle \in \partial_{a}(S)\right\}$ is exactly the Antimirov derivative of $R$ for $a$. The Antimirov construction has also been generalized to extended regexes [Caron et al. 2011] allowing Boolean operators such as complement and intersection. Basic theoretical properties between various automata formalisms and derivatives are discussed in [Allauzen and Mohri 2006].

Automata with counting. This work is a continuation of our recent work [Holík et al. 2019]. In [Holík et al. 2019], we propose a general determinization of CAs that can produce smaller automata than the naive explicit determinization but has the same worst-case complexity, which depends on the counters with the factor $(K+1)^{|C|}$ where $C$ is the set of counters and $K$ the maximum counter upper bound. It also proposes a more efficient algorithm for the class of monadic regexes 
(single-state-scoped counters and counting on self-loops only), but it can still generate $(K+1)^{|Q|}$ states (e.g., it would generate $K+1$ states for the regex from Fig. 1)-while the complexity of our determinization does not depend on $K$. The work [Holík et al. 2019] does also not present a derivative construction for translating regexes into CAs nor an application of CAs in pattern matching.

The use of counters has also been investigated in [Björklund et al. 2015] for regexes with bounded repetition, building on the formalism of counter automata called CNFAs [Gelade et al. 2012]. A CA in the current paper is essentially a symbolic generalization of a CNFA with some small technical differences, such as counters being 0-based as opposed to 1-based in a CNFA. The latter difference is mainly due to our use of a generalized Antimirov construction of CAs, as opposed to a generalized Glushkov construction used in [Gelade et al. 2012], which is algorithmically quite different. The work in [Björklund et al. 2015] focuses mostly on deterministic regexes and on a different problem, namely, the so-called incremental matching in the context of database queries (a query is repeatedly evaluated on a gradually changing word). For standard matching, it uses a variant of the Thompson's algorithm applied directly on a CA instead of an NFA (hence the translation of the regex to an automaton does not depend on the counter bounds, but each text character is processed with the same cost as with the original Thompson's algorithm, at worst linear to the size of the NFA and the counter bounds). This algorithm is indeed fast on deterministic regexes from practice but can slow down significantly on nondeterministic ones (which we witnessed in several experiments with the prototype implementation of [Björklund et al. 2015] on several of our regexes).

The work in [Kilpeläinen and Tuhkanen 2003] is a theoretical study of matching regexes with counting. It proposes a matching algorithm based on dynamic programming that runs in time at worst quadratic to the length of the text (while determinization and NFA-simulation-based algorithms run in time linear to the text length). The experimental comparison of [Björklund et al. 2015] with their variant of Thompson's algorithm suggests that the matching algorithm of [Kilpeläinen and Tuhkanen 2003] is indeed not competitive in practice.

Extended FAs (XFAs) augment classical automata with a scratch memory of bits [Smith et al. 2008a,b] that can represent counters. Regexes are compiled into deterministic XFAs by first using an extended version of the Thompson's algorithm, followed by an extended version of the classical powerset construction and minimization. Although a small XFA may exist, the determinization algorithm incurs an intermediate exponential blowup of the search space for inputs such as $\bullet * a .\{k\}$.

$R$-automata [Abdulla et al. 2008] are also related to our CAs, but their counters need not have upper bounds and cannot be tested or compared. Further, there are various notions of extended finite state machines whose expressive power goes beyond regular languages, e.g., [Bardin et al. 2008; Cheng and Krishnakumar 1993; Shiple et al. 1998; Smith et al. 2008b]. Such automata are, however, not suitable for the problem of pattern matching considered here.

Regexes with counting. Regexes with counters are also discussed in [Gelade et al. 2007; Hovland 2009; Kilpeläinen and Tuhkanen 2007]. The automata with counters used in [Hovland 2009], called FACs, are close to our CAs, but we allow symbolic character predicates and more kinds of counter updates. The conversion from regexes to FACs proposed in [Hovland 2009] uses a variant of Glushkov automata [Glushkov 1961] and the first-last-follow construction [Berstel and Pin 1996; Brüggemann-Klein and Wood 1998]. For us, the Antimirov-derivative-based construction was easier to implement and provides benefits that are not available otherwise. Namely, it allows subsumption checking between regexes, and it generates fewer counters (one per distinct counter sub-expression rather than one per counter position in the regex abstract syntax tree). While all these algorithms generate $\epsilon$-free automata, they differ in complexity [Allauzen and Mohri 2006] and are thus not merely different disguises of the same technique. In particular, the Antimirov automaton is in general smaller than the Glushkov automaton with up to $n+1$ states and up to $n^{2}$ transitions. 
The Antimirov automaton is in fact a quotient of the Glushkov automaton [Champarnaud and Ziadi 2001; Ilie and Yu 2003]. Another generalization of Antimirov derivatives [Lombardy and Sakarovitch 2005] introduces expressions $k R$ where $R$ is a rational expression and $k$ a multiplicity from a semiring such as $\mathbb{Q}$; this generalization is unrelated to counters.

An open question is whether the generalized Antimirov construction can be extended to work with Brzozowski derivatives [Brzozowski 1964]; we believe that such an extension, if it exists, is not straightforward because it would give rise to a direct and incremental determinization algorithm.

There are also works on regexes with counting that translate deterministic regexes to CAs and work with different notions of determinism [Chen and Lu 2015; Gelade et al. 2012]. A central result in [Hovland 2009] is that counter-1-unambiguous regexes can be compiled into deterministic FACs and that checking determinism of FACs can be done in polynomial time. The related work in [Hovland 2012] studies membership in regexes with counting. None of these papers addresses the problem of determinizing nondeterministic CAs.

Pattern matching of regexes with counting. The counting operator often appears in regexes in practice. In particular, our analysis of the 537k real-world regexes obtained in the study performed by Davis et al. [Davis et al. 2019] showed that over 33k regexes contained the counting operator.

GNU grep [Haertel et al. [n.d.]] (written in C) and RE2 [Google [n.d.]] (written in C++) are extremely optimized regex matchers. Both are based on translating the regex into an NFA and performing an on-the-fly determinization during the matching, avoiding a costly a priori determinization, while keeping a good performance by avoiding backtracking. (The translation into FAs is only allowed when the regex does not include back-references, which allow one to express some context-free properties). Both engines process the counting operator by first rewriting a regex of the form $\langle$ re $>\{n, m\}$ into $\langle$ re $>. .\langle$ re $>\langle$ re $>\{0, m-n\}$. The regex $\langle$ re $>\{\theta, k\}$ is then transformed into $(\langle\mathrm{re}\rangle(\langle\mathrm{re}\rangle(\ldots<\mathrm{re}\rangle$ ?) ?) ?) ? (see [Cox 2010] for more details).

In the .NET ecosystem, we are aware of two regex matchers. The first one is the standard .NET regex matcher provided in System. Text.RegularExpressions, which is based on a backtracking search. The other one is Symbolic Regex Matcher (SRM) of [Saarikivi et al. 2019] based on the socalled symbolic derivatives, which provide a backtracking-free search (without an explicit conversion into a DFA) and can deal more efficiently with the counting operator.

\section{CONCLUSIONS AND FUTURE WORK}

We have presented a framework for efficient pattern matching of regexes with counting, which includes a derivative construction to compile regexes to counting automata, their subsequent determinization into novel counting-set automata, and a fast matching algorithm. The resources needed to build the CsAs are independent of counter bounds. It handles a majority of regexes with counting found in practice, with a much more stable performance than other matchers.

In the future, we intend to explore the limits of the idea of counting sets to enlarge and clearly delimit the class of regexes and counting automata that can be succinctly determinized while preserving fast matching. We also plan to explore possible usage of CsAs as a replacement of classical automata in other applications where automata are used, for instance, as symbolic representations of state spaces. For this, we intend to develop CsA counterparts of essential automata techniques, such as Boolean operations and minimization/size-reduction techniques. We also wish to elaborate on our method for generating texts for stress-testing matchers on regexes with counting.

\section{ACKNOWLEDGMENTS}

We thank the anonymous reviewers and also Juraj Síč for their valuable comments and suggestions. This work is supported by the Czech Ministry of Education, Youth and Sports project LL1908 of the ERC.CZ programme, and the FIT BUT internal project FIT-S-20-6427. 


\section{REFERENCES}

Parosh Aziz Abdulla, Pavel Krčál, and Wang Yi. 2008. R-Automata. In CONCUR'08 (LNCS, Vol. 5201). Springer, 67-81. https://doi.org/10.1007/978-3-540-85361-9_9

Cyril Allauzen and Mehryar Mohri. 2006. A Unified Construction of the Glushkov, Follow, and Antimirov Automata. In Mathematical Foundations of Computer Science 2006. Springer Berlin Heidelberg, Berlin, Heidelberg, 110-121. https: //doi.org/10.1007/11821069_10

Valentin Antimirov. 1996. Partial derivatives of regular expressions and finite automaton constructions. Theoretical Computer Science 155, 2 (1996), 291 - 319. https://doi.org/10.1016/0304-3975(95)00182-4

Adam Baldwin. 2016. Regular Expression Denial of Service affecting Express.js. http://web.archive.org/web/20170116160113/ https://medium.com/node-security/regular-expression-denial-of-service-affecting-express-js-9c397c164c43

Sébastien Bardin, Alain Finkel, Jérôme Leroux, and Laure Petrucci. 2008. FAST: acceleration from theory to practice. STTT 10, 5 (2008), 401-424. https://doi.org/10.1007/s10009-008-0064-3

Gerard Berry and Ravi Sethi. 1986. From regular expressions to deterministic automata. Theoretical Computer Science 48, 3 (1986), 117-126. https://doi.org/10.1016/0304-3975(86)90088-5

Jean Berstel and Jean-Éric Pin. 1996. Local languages and the Berry-Sethi algorithm. Theoret. Comput. Sci. 155, 2 (1996), 439-446. https://doi.org/10.1016/0304-3975(95)00104-2

Henrik Björklund, Wim Martens, and Thomas Timm. 2015. Efficient Incremental Evaluation of Succinct Regular Expressions. In CIKM'15 (ACM). https://doi.org/10.1145/2806416.2806434

Robert S. Boyer and J. Strother Moore. 1977. A Fast String Searching Algorithm. Commun. ACM 20, 10 (Oct. 1977), 762-772. https://doi.org/10.1145/359842.359859

Anne Brüggemann-Klein and Derick Wood. 1998. One-unambiguous regular languages. Information and Computation 140, 2 (1998), 229-253. https://doi.org/10.1006/inco.1997.2695

Janusz A. Brzozowski. 1964. Derivatives of Regular Expressions. F. ACM 11, 4 (1964), 481-494. https://doi.org/10.1145/ 321239.321249

Pascal Caron, Jean-Marc Champarnaud, and Ludovic Mignot. 2011. Partial Derivatives of an Extended Regular Expression. In Language and Automata Theory and Applications. Springer Berlin Heidelberg, Berlin, Heidelberg, 179-191. https: //doi.org/10.1007/978-3-642-21254-3_13

Jean-Marc Champarnaud and Djelloul Ziadi. 2001. Computing the equation automaton of a regular expression in $O\left(s^{2}\right)$ space and time. In Proceedings of CPM 2001 (LNCS, Vol. 2089). Springer, 157-168. https://doi.org/10.1007/3-540-48194-X_15

Haiming Chen and Ping Lu. 2015. Checking determinism of regular expressions with counting. Information and Computation 241 (2015), 302 - 320. https://doi.org/10.1016/j.ic.2014.12.001

Kwang-Ting Cheng and A. S. Krishnakumar. 1993. Automatic Functional Test Generation Using the Extended Finite State Machine Model. In Proceedings of the 30th Design Automation Conference. Dallas, Texas, USA, fune 14-18, 1993. ACM Press, 86-91. https://doi.org/10.1145/157485.164585

Wikipedia contributors. 2019. Regular expression-Wikipedia. https://en.wikipedia.org/w/index.php?title=Regular_ expression\&\%20oldid $=852858998$

Russ Cox. 2010. Regular Expression Matching in the Wild. https://swtch.com/ rsc/regexp/regexp3.html.

Loris D'Antoni and Margus Veanes. 2020. Automata Modulo Theories. Commun. ACM (2020).

James C. Davis. 2019. Rethinking Regex Engines to Address ReDoS. In Proceedings of ESEC/FSE'19 (Tallinn, Estonia) (ESEC/FSE 2019). ACM, New York, NY, USA, 1256-1258. https://doi.org/10.1145/3338906.3342509

James C. Davis, Christy A. Coghlan, Francisco Servant, and Dongyoon Lee. 2018. The Impact of Regular Expression Denial of Service (ReDoS) in Practice: An Empirical Study at the Ecosystem Scale. In Proceedings of ESEC/FSE'18 (Lake Buena Vista, FL, USA) (ESEC/FSE 2018). ACM, New York, NY, USA, 246-256. https://doi.org/10.1145/3236024.3236027

James C. Davis, Louis G. Michael IV, Christy A. Coghlan, Francisco Servant, and Dongyoon Lee. 2019. Why Aren't Regular Expressions a Lingua Franca? An Empirical Study on the Re-use and Portability of Regular Expressions. In Proceedings of ESEC/FSE'19 (Tallinn, Estonia) (ESEC/FSE 2019). ACM, New York, NY, USA, 1256-1258. https://doi.org/10.1145/3338906. 3338909

Stack Exchange. 2016. Outage Postmortem. http://stackstatus.net/post/147710624694/outage-postmortem-july-20-2016

Sebastian Fischer, Frank Huch, and Thomas Wilke. 2010. A Play on Regular Expressions: Functional Pearl. SIGPLAN Not. 45, 9 (2010), 357-368. https://doi.org/10.1145/1863543.1863594

Wouter Gelade, Marc Gyssens, and Wim Martens. 2012. Regular Expressions with Counting: Weak versus Strong Determinism. SIAM f. Comput. 41, 1 (2012), 160-190. https://doi.org/10.1137/100814196 Extended version of paper in MFCS'09.

Wouter Gelade, Wim Martens, and Frank Neven. 2007. Optimizing schema languages for XML: Numerical constraints and interleaving. In Proceedings of ICDT'07 (LNCS, Vol. 4353). Springer, 269-283. https://doi.org/10.1007/11965893_19

V. M. Glushkov. 1961. The abstract theory of automata. Russian Math. Surveys 16 (1961), 1-53. https://doi.org/10.1070/ RM1961v016n05ABEH004112

Proc. ACM Program. Lang., Vol. 4, No. OOPSLA, Article 218. Publication date: November 2020. 
Google. [n.d.]. RE2. https://github.com/google/re2.

John Graham-Cumming. 2019. Details of the Cloudflare outage on fuly 2, 2019. https://blog.cloudflare.com/details-of-thecloudflare-outage-on-july-2-2019/

Mike Haertel. [n.d.]. why GNU grep is fast. https://ists.freebsd.org/pipermail/freebsd-current/2010-August/019310.html.

Mike Haertel et al. [n.d.]. GNU grep. https://www.gnu.org/software/grep/.

Lukáš Holík, Ondřej Lengál, Olli Saarikivi, Lenka Turoňová, Margus Veanes, and Tomáš Vojnar. 2019. Succinct Determinisation of Counting Automata via Sphere Construction. In Proc. of APLAS'19 (LNCS, Vol. 11893). Springer, 468-489. https://doi.org/10.1007/978-3-030-34175-6_24

Lukáš Holík, Ondřej Lengál, Olli Saarikivi, Lenka Turoňová, Margus Veanes, and Tomáš Vojnar. 2020. Dataset for the OOPSLA'20 paper "Regex Matching with Counting-Set Automata". https://doi.org/10.5281/zenodo.3974360

Dag Hovland. 2009. Regular Expressions with Numerical Constraints and Automata with Counters. In ICTAC (LNCS, Vol. 5684). Springer, 231-245. https://doi.org/10.1007/978-3-642-03466-4_15

Dag Hovland. 2012. The Membership Problem for Regular Expressions with Unordered Concatenation and Numerical Constraints. In Language and Automata Theory and Applications. Springer Berlin Heidelberg, Berlin, Heidelberg, 313-324. https://doi.org/10.1007/978-3-642-28332-1_27

Lucian Ilie and Sheng Yu. 2003. Follow automata. Information and Computation 186, 1 (2003), 146-162. https://doi.org/10. 1016/S0890-5401(03)00090-7

Pekka Kilpeläinen and Rauno Tuhkanen. 2003. Regular Expressions with Numerical Occurrence Indicators - preliminary results. In Proceedings of the Eighth Symposium on Programming Languages and Software Tools, SPLST'03, Kuopio, Finland, fune 17-18, 2003. University of Kuopio, Department of Computer Science, 163-173.

Pekka Kilpeläinen and Rauno Tuhkanen. 2007. One-unambiguity of regular expressions with numeric occurrence indicators. Information and Computation 205, 6 (2007), 890-916. https://doi.org/10.1016/j.ic.2006.12.003

Sylvain Lombardy and Jacques Sakarovitch. 2005. Derivatives of rational expressions with multiplicity. Theoretical Computer Science 332, 1 (2005), 141 - 177. https://doi.org/10.1016/j.tcs.2004.10.016

M. Roesch et al. [n.d.]. Snort: A Network Intrusion Detection and Prevention System,. http://www.snort.org.

Microsoft. 2020. . https://docs.microsoft.com/en-us/dotnet/api/system.text.regularexpressions.regex.match

Scott Owens, John Reppy, and Aaron Turon. 2009. Regular-expression Derivatives Re-examined. f. Funct. Program. 19, 2 (2009), 173-190. https://doi.org/10.1017/S0956796808007090

Mono project. [n.d.]. Mono. https://www.mono-project.com/.

RegExLib.com. [n.d.]. The Internet's first Regular Expression Library, . http://regexlib.com/.

Robin Sommer et al. [n.d.]. The Bro Network Security Monitor. http://www.bro.org.

Olli Saarikivi, Margus Veanes, Tiki Wan, and Eric Xu. 2019. Symbolic Regex Matcher. In TACAS’2019 (LNCS, Vol. 11427), Tomás Vojnar and Lijun Zhang (Eds.). Springer, 372-378. https://doi.org/10.1007/978-3-030-17462-0_24

Thomas R. Shiple, James H. Kukula, and Rajeev K. Ranjan. 1998. A Comparison of Presburger Engines for EFSM Reachability. In Computer Aided Verification, 10th International Conference, CAV'98, Vancouver, BC, Canada, June 28 - fuly 2, 1998, Proceedings (Lecture Notes in Computer Science, Vol. 1427). Springer, 280-292. https://doi.org/10.1007/BFb0028752

Michael Sipser. 2006. Introduction to Theory of Computation. Vol. 2. Thomson Course Technology Boston. https://doi.org/10. 1145/230514.571645

Randy Smith, Cristian Estan, and Somesh Jha. 2008a. XFA: Faster Signature Matching with Extended Automata. In IEEE Symposium on Security and Privacy. IEEE. https://doi.org/10.1109/SP.2008.14

Randy Smith, Cristian Estan, Somesh Jha, and Ida Siahaan. 2008b. Fast Signature Matching Using Extended Finite Automaton (XFA). In ICISS'08 (LNCS, Vol. 5352). Springer, 158-172. https://doi.org/10.1007/978-3-540-89862-7_15

Henry Spencer. 1994. Software Solutions in C. Academic Press Professional, Inc., San Diego, CA, USA, Chapter A Regularexpression Matcher, 35-71. http://dl.acm.org/citation.cfm?id=156626.184689

Michael Sperberg-McQueen. [n.d.]. Notes on finite state automata with counters. https://www.w3.org/XML/2004/05/msmcfa.html Accessed: 2018-08-08.

The Sagan team. [n.d.]. The Sagan Log Analysis Engine. https://quadrantsec.com/sagan_log_analysis_engine/.

Ken Thompson. 1968. Programming Techniques: Regular Expression Search Algorithm. Commun. ACM 11, 6 (June 1968), 419-422. https://doi.org/10.1145/363347.363387

Lenka Turoňová, Lukáš Holík, Ondřej Lengál, Olli Saarikivi, Margus Veanes, and Tomáš Vojnar. [n.d.]. Automata library. https://pajda.fit.vutbr.cz/ituronova/countingautomata.

Lenka Turoňová, Lukáš Holík, Ondřej Lengál, Olli Saarikivi, Margus Veanes, and Tomáš Vojnar. 2020. Regex Matching with Counting-Set Automata. Technical Report MSR-TR-2020-31. Microsoft. https://doi.org/10.5281/zenodo.3975566

Milan Češka, Vojtěch Havlena, Lukáš Holík, Ondřej Lengál, and Tomáš Vojnar. 2018. Approximate Reduction of Finite Automata for High-Speed Network Intrusion Detection. In Proc. of TACAS’18 (LNCS, Vol. 10806). Springer. https: //doi.org/10.1007/978-3-319-89963-3_9 
Margus Veanes, Peli de Halleux, and Nikolai Tillmann. 2010. Rex: Symbolic Regular Expression Explorer. In Third International Conference on Software Testing, Verification and Validation, ICST 2010, Paris, France, April 7-9, 2010. 498-507. https: //doi.org/10.1109/ICST.2010.15

Liu Yang, Rezwana Karim, Vinod Ganapathy, and Randy Smith. 2010. Improving NFA-Based Signature Matching Using Ordered Binary Decision Diagrams. In Recent Advances in Intrusion Detection. Springer Berlin Heidelberg, Berlin, Heidelberg, 58-78. https://doi.org/10.1007/978-3-642-15512-3_4 